\title{
Three-dimensional evolution of flow structures in transitional circular and chevron jets
}

\author{
Daniele Violato ${ }^{\text {a) }}$ and Fulvio Scarano \\ Department of Aerospace Engineering, Delft University of Technology, Kluyverweg 1, 2629 HS, Delft, \\ The Netherlands
}

(Received 10 May 2011; accepted 3 November 2011; published online 27 December 2011)

\begin{abstract}
The three-dimensional behavior of flow transition in circular and 6-chevron jets at $R e=5000$ is investigated with experiments conducted on a free water jet by time-resolved tomographic particle image velocimetry. The emphasis is on the unsteady organization of coherent flow structures, which play a role in the generation of acoustic noise. Shedding and pairing of vortices are the most pronounced phenomena observed in the near field of the circular jet. The first and second pairing amplify the axial pulsatile motion in the jet column and lead to the growth of azimuthal waves culminating in the breakup of the vortex ring. Streamwise vortices of axial and radial vorticity are observed in the outer region and move inward and outward under the effect of the vortex rings. In the jet with chevrons, the axisymmetric ring-like coherence of the circular jet is not encountered. Instead, streamwise flow structures of azimuthal vorticity emanate from the chevron apices, and counter-rotating streamwise vortices of axial and radial vorticity develop from the chevron notches. The decay of streamwise vortices is accompanied by the formation of $\mathrm{C}$-shaped structures. The three-dimensional analysis allows quantifying the vortex stretching and tilting activity, which, for the circular jet exit, is related to the azimuthal instabilities and the streamwise vortices connecting the vortex rings. In the chevron jet, stretching and tilting peak during the formation of C-structures. Following Powell's aeroacoustic analogy, the spatial distribution of the source term is mapped, evaluating the temporal derivative of the Lamb vector. The spatio-temporal evolution of such source term is visualized revealing that the events of highest activity are associated with the processes of vortex-ring pairing and vortex-ring disruption for the circular jet, and with the decay of streamwise instabilities and the formation of C-shaped structures for the chevron case. (C) 2011 American Institute of Physics. [doi:10.1063/1.3665141]
\end{abstract}

\section{INTRODUCTION}

The process of flow transition occurring in jets with laminar exit is governed by the growth of unstable modes introducing large-scale coherent flow structures. The transition pattern is reported to largely depend upon the geometry of the nozzle exit, Reynolds number, and the nozzle exit velocity profile. In many engineering applications, passive mixing- and acoustic-control strategies are based on the alterations of the jet exit geometry. Compared to the circular jet, the mean entrainment rate increases in elliptic ${ }^{1}$ and lobed jets, ${ }^{2}$ while reduced acoustic emissions are characteristic of chevron jets. ${ }^{3}$ The dynamical behavior of large-scale structures and their interactions in jets in the compressible regime are particularly relevant to aeroacoustics. However, the fundamental physical processes at the origin of sound generation are associated with the flow turbulence and its complex three-dimensional nature with energy distributed over a wide spectrum.

The role of large-scale flow structures in jet flows can be studied with the direct numerical simulation (DNS) and large eddy simulation (LES) techniques, ${ }^{4,5}$ which offer a formidable tool for the detailed analysis of unsteady flow behavior. More complex geometries such as lobed and chevron nozzles

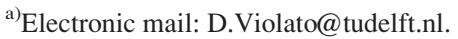

at high Reynolds numbers have been recently afforded with LES, ${ }^{6,7}$ whereas investigations with DNS have mostly focused on the fundamental aspects of circular jets at low Reynolds number. ${ }^{4}$

Experimental investigations on the organization of coherent flow structures require field measurement techniques such as flow visualization ${ }^{8-11}$ and, in the last decades, particle image velocimetry (PIV). PIV measurements are typically conducted along streamwise or cross-sectional planes to investigate the unsteady behavior of the shear layer and the transition patterns. ${ }^{12,13}$ Cross sectional measurements perpendicular to the jet axis enabled a quasi-3D representation of the turbulent coherent structures invoking Taylor's hypothesis in the fully developed turbulent region. ${ }^{14}$ A recently developed high speed three-dimensional flow visualization technique combined with proper orthogonal decomposition (POD) ${ }^{15}$ showed that vortex rings, azimuthal ring waves, and streamwise vortex structure are formed in circular jets at Reynolds number of 10000 . However, the corresponding vorticity pattern remains to be ascertained by $3 \mathrm{D}$ velocimetry techniques.

The description of the dynamical behavior and the 3D patterns of flow structures requires time-resolved, volumebased techniques. Time resolved PIV measurements were conducted by Wernet ${ }^{16}$ on high-speed jet to characterize the turbulence by means of space-time correlation functions. The 
first instantaneous 3D vorticity field of a jet at $R e=1000$ was performed by Hori and Sakakibara, ${ }^{17}$ based on a high speed scanning stereo PIV system. ${ }^{18}$ The development of tomographic PIV (TOMO-PIV) ${ }^{19}$ enables the measurement of the instantaneous velocity vector field over a three-dimensional domain, with the advantage that the velocity and the complete velocity gradient tensor and the associated quantities (e.g., the vorticity vector and the second invariant) can be obtained independently of the flow speed. In jet flow studies, the TOMO PIV technique has been successfully applied to explore the topology of a turbulent interface ${ }^{20}$ and was demonstrated by Staack et $a .^{21}$ to yield more accurate results than the scanning light sheet technique, according to the compliance to flow incompressibility. High-speed TOMO-PIV is adopted in the present investigation to yield high temporal and spatial resolution for the study of the unsteady 3D behavior of flow transition in circular and chevron jets.

The role of large-scale coherent structures in circular jets has been the objective of several investigations. In the region next to the nozzle, the fluctuations are organized as toroidal vortices generated by the growth of the KelvinHelmholtz (K-H) instability. ${ }^{8,22}$ At moderate Reynolds number, such structures grow maintaining axial symmetry and undergo pairing due to the mutual flow induction. ${ }^{9,23-25}$ Further downstream, the flow transition to turbulence occurs with the growth of three-dimensional instabilities, such as azimuthal oscillations of the vortex rings. ${ }^{12,26,27}$ Counterrotating pairs of streamwise vortices are also reported to exist in the braid region between two vortex rings. ${ }^{10-13,15}$

Modifying the shape of the jet exit geometry has dramatic effects on the organization and stability of the shear layer with consequences on the entrainment rate, the spectral characteristics, and the related acoustic emissions. Early investigations from Paterson ${ }^{28}$ showed that nozzles with a lobed exit generate coherent streamwise vortices emanating from the exit. Ho and Gutmark ${ }^{1}$ observed that an elliptic nozzle sheds vortices with pre-defined azimuthal distortion, producing an enhancement of the mean entrainment rate. Zaman and Raman ${ }^{29}$ placed tabs at the exit of turbulent circular jet reporting a marked loss of azimuthal coherence and the generation of pairs of streamwise vortices, which contribute to the increase of the lateral spreading of the jet along the direction orthogonal to the plane of the tabs. Streamwise structures are also formed in rectangular jets, as shown by the phased-locked stereo PIV measurements by Alkislar et al. ${ }^{30}$ More recently, El Hassan and Meslem ${ }^{2}$ studied the entrainment of circular and daisy-shaped orifice jets at $R e=3600$ using time-resolved stereo PIV and snapshot POD analysis. They concluded that the daisy-shaped orifice jet undergoes earlier transition due to the generation of streamwise vortices, with a significant enhancement of the entrainment process. A similar methodology was adopted by El Hassan et al., ${ }^{31}$ who reported the influence of K-H structures on the instantaneous entrainment and net volume flux.

The idea that coherent flow structures of the jet near region are involved in the sound generation mechanism $^{9,32-35}$ suggests that a reduction of the flow azimuthal coherence, for instance by using chevrons, could promote local cancellation of large-scale acoustic fluctuations, thus leading to far-field acoustic suppression. ${ }^{22}$ In a survey on chevron nozzles, Bridges and Brown ${ }^{3}$ used PIV and microphone measurements to investigate the relationship between flow-field patterns, far field noise, and chevron geometric parameters. From the time-averaged properties, it emerged clearly that the chevron penetration depth influences the axial vorticity acting on the shear layer and, ultimately, leading to a faster decay of the core velocity. Furthermore, Opalski et al $^{36}{ }^{3}$ investigated the relationship between the chevron geometry and flow characteristics, reporting extensive flow statistics at Mach numbers ranging between 0.9 and 1.5, for both cold and hot jets. Stereoscopic PIV was used to measure the three components of the mean and fluctuating velocity, as well as the axial vorticity and turbulent kinetic energy, over a region extending beyond 20 nozzle diameters downstream the nozzle exit, reporting the presence of well-defined streamwise vortices. The relation between the latter and the reduction of low-frequency acoustic emissions of 9-chevron configuration (Mach 0.9, $R e=1.3 \times 10^{6}$ ) was investigated by Alkislar et al. ${ }^{37}$ who used stereoscopic PIV and microphone measurements. Numerical studies making use of Reynolds-averaged NavierStokes (RANS) calculations in the near field have been performed by Thomas et $a l .{ }^{38}$ and Birch et al. ${ }^{39}$ The latter showed that the chevrons generate axial vortices increase the mixing layer growth rate.

The experimental investigations of chevron jets have mostly focused on flow statistics with larger emphasis on the acoustic emissions rather than the description of instantaneous flow patterns. Nevertheless, as the acoustic production is strongly related to the large-scale flow motion, ${ }^{32,33,40}$ the observation of the temporal evolution of large-scales structures is of major importance to understand the physical mechanism behind the production of acoustic noise.

In the last decade, PIV has been successfully used in combination with aeroacoustic analogies to study the flowfield pattern producing noise. In a Mach 0.85 jet, Seiner et $a l .{ }^{41}$ characterized the noise sources using two-point turbulence statistics to evaluate the Lighthill turbulent stress tensor, concluding that the actual measurement of the Lighthill's acoustic tensor requires volumetric measurements with temporal resolution. In a low-speed acoustically excited jet, Schram et al. ${ }^{42}$ investigated the mechanism of sound generation by vortex pairing with phase-locked planar PIV and a conservative formulation of vortex sound theory for axisymmetric flow, finding good agreement between the sound prediction and the sound measured by microphone at frequencies not contaminated by the acoustical excitation. Fleury et al $^{43}$ used a dual-PIV system in a subsonic jet at Mach 0.6 and 0.9 with a fixed time delay between measurements to calculate spatial and temporal correlations. They also showed that Lighthill stress tensor can be statistically modeled by space-time second-order velocity correlations. Recalling Howe's acoustic analogy, Henning et al. ${ }^{44}$ performed planar PIV and phased-microphone-array measurements and investigated the acoustic source in the vicinity of a circular cylinder and an airfoil leading-edge slat by crosscorrelation function between the acoustic pressure and velocity/vorticity fluctuations. In cavity flows, Haigermoser, ${ }^{45}$ and 
later Koschatzky et al., ${ }^{46}$ reconstructed the pressure field from time-resolved planar PIV and then predicted far-field acoustics by Curle's analogy using the pressure information on the cavity surface. The potential and relevance of PIV studies in aeroacoustics have been acknowledged in a recent review article by Morris, ${ }^{47}$ where the role of time-resolved PIV was emphasized. In this respect, the tomographic variant provides a more complete approach to obtain information about the sound generation.

The main questions driving the need for TR-TOMO PIV are to what extent the nozzle geometry affects the instantaneous azimuthal coherence and what is the role of the instantaneous coherent fluctuations exhibited during the transition to the turbulent regime. Given the three-dimensional complex pattern of the flow, the above questions require a full 3D approach to produce an unambiguous description of the vortex topology. Moreover, the temporal resolution of the phenomenon allows addressing the growth and development of the coherent flow structures along with their mutual interaction. TR-TOMO PIV is a suitable technique for the diagnostics of complex three-dimensional flows, as shown by recent applications on turbulent boundary layers ${ }^{48,49}$ and wake flows. ${ }^{50,51}$ The application to circular and chevron jets has been demonstrated feasible in the visualizations obtained by Scarano et $a l .{ }^{52}$ The use of time-resolved experiments to determine the sound produced by the early transition stage due to vortex pairing ${ }^{42}$ has been extended to the natural transition case by Violato et al. ${ }^{53}$ With direct numerical simulations tailored on the latter experiment, Moore et al. ${ }^{54}$ showed that the experimental approach is also a valuable tool to provide a comparison based on unsteady flow properties to numerical simulations.

The objective of this study is to investigate the impact of three-dimensional disturbances on the entire transition pattern in jets at moderate Reynolds number, with a comparative analysis between the circular and chevron configuration. For the circular jet, the emphasis is placed upon the dynamical evolution of the coherent vortex structures and their interaction in the transition from the axisymmetric to the three-dimensional regime.

The chosen 6-chevron jet configuration follows that proposed by Bridges and Brown, ${ }^{3}$ who performed experiments at considerably higher Reynolds number. Despite not enabling quantitative comparisons with the work of Bridges and Brown, ${ }^{3}$ the current investigation is intended to clarify some fundamental processes occurring in the transition to turbulence. For this purpose, the investigation is conducted under favorable experimental conditions in a water facility, where a relatively large measurement domain can be accessed with TOMO-PIV and where high-speed PIV systems can operate at a repetition rate considerably higher than the temporal resolution needed to describe the fastest flow events.

The work presents first an analysis of the vortex topology for the circular and chevron jets using the vorticity and the $\lambda_{2}$-criterion. ${ }^{55}$ Additional information to the large bulk of existing knowledge on the vortex-dominated events is discussed, providing a quantitative analysis of vortex properties (e.g., vortex strength and stretching-tilting activity), which are of difficult evaluation by other experimental techniques.
Moreover, the integrand of Powell's acoustic analogy ${ }^{56}$ is evaluated from the velocity field measurements, and the spatial distribution of the noise source is associated with the second time derivative of the Lamb vector. This is visually inspected and the regions of most activity are finally characterized on a statistical basis.

\section{EXPERIMENTAL APPARATUS AND PROCEDURES}

\section{A. Jet flow facility}

Experiments are conducted in a water facility at the Aerodynamic Laboratories of TU Delft in the Aerospace Engineering Department. A round nozzle of exit diameter $D=10 \mathrm{~mm}$ and contraction ratio of $56: 1$ is installed at bottom wall of a tank. The nozzle contraction is shaped following the work of Schram et al. ${ }^{42}$ that yields a uniform laminar flow at the exit. The jet is enclosed in an octagonal water tank of $600 \mathrm{~mm}$ diameter and $800 \mathrm{~mm}$ height built in Plexiglas to enable extensive optical access for illumination and tomographic imaging (Figure 1).

The 6-chevrons jet exit is applied on top of the circular nozzle profile, with chevrons $7 \mathrm{~mm}$ high and inclined towards the jet axis by an angle to the jet axis of $12^{\circ}$, as shown in Figure 2 , thus resulting in a penetration depth of $1.5 \mathrm{~mm}$.

The system is driven hydrostatically and provides a stabilized supply in a range of exit velocity $W_{j}$ from 0.1 to $2 \mathrm{~m} / \mathrm{s}$, corresponding to Reynolds numbers ranging between 1000 and 20000. Tomographic experiments are performed for a nominal axial velocity at the jet exit of $0.5 \mathrm{~m} / \mathrm{s}$ yielding a Reynolds number $R e=5000$ based on the jet diameter $D$. Additionally, planar PIV experiments are conducted on the circular jet for statistical characterization of the flow.

\section{B. Time-resolved tomographic measurements}

Neutrally buoyant polyamide particles of $56 \mu \mathrm{m}$ of diameter are dispersed homogeneously, achieving a uniform

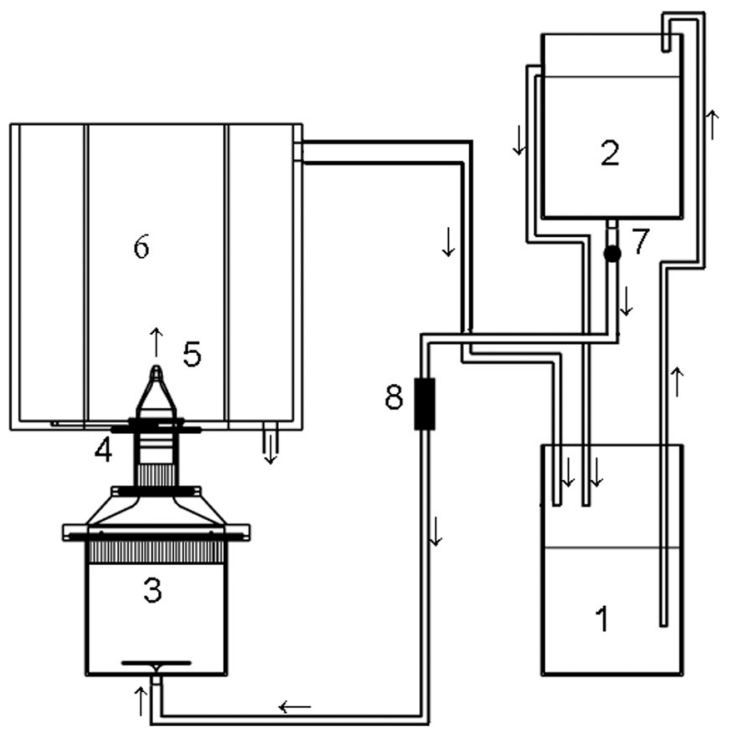

FIG. 1. Jet tomographic facility: a schematic of the system; (1) water reservoir, (2) piezometric water tank, (3) jet tank, (4) settling chamber, (5) nozzle, (6) Plexiglas water tank, (7) valve, (8) flow meter. 


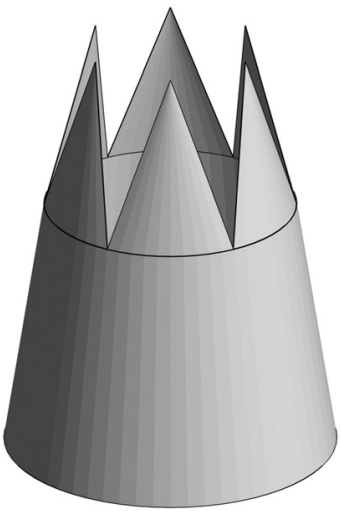

FIG. 2. Chevron nozzle.

concentration of 0.65 particles $/ \mathrm{mm}^{3}$. The illumination is provided by a Quantronix Darwin-Duo solid-state diodepumped Nd:YLF (neodymium: yttrium lithium fluoride) laser $(2 \times 25 \mathrm{~mJ} /$ pulse at $1 \mathrm{kHz})$. The laser beam after a transmission distance of $1.5 \mathrm{~m}$ reaches a diameter of $6 \mathrm{~mm}$, increased to $30 \mathrm{~mm}$ with a beam expander and directed towards the nozzle vertically (Figure 3). The light scattered by the particles is recorded by a tomographic system composed of four Lavision HighSpeedStar 6 CMOS (complementary metal-oxide-semiconductor) cameras $(1024 \times 1024$ pixels, 5400 frames/s, pixel pitch $20 \mu \mathrm{m}$ ) arranged horizontally with azimuthal aperture of $90^{\circ}$. The choice of illuminated volume with a beam of cylindrical cross section eliminates the need for camera-lens tilt mechanism to comply with the Scheimpflug condition. Nikon objectives of $105 \mathrm{~mm}$ focal length are set with a numerical aperture $f_{\#}=32$ to allow focused imaging of the illuminated particles. For the chosen illumination and imaging configuration, the particle image density attains a maximum of 0.04 particles/ pixel at the jet axis and decreases towards the edge of the illuminated volume. The details of the experimental settings are summarized in Table I.
The choice of a cylindrical domain of illumination also results in a more favorable condition for accurate reconstruction of the particle field, as the particle image density does not change with the viewing angle along the azimuth and decreases moving from the axis to the periphery of the jet (Figure 3).

Sequences of images of tracer particles (Figure 4) are recorded at $1 \mathrm{kHz}$ resulting in a temporal resolution that yields 35 samples for the fastest expected events (vortex shedding). The imaged-particle displacement at the exit is approximately 8 pixels along the jet axis. The field of view is of $50 \times 50 \mathrm{~mm}$ with a digital resolution of 21.5 pixels $/ \mathrm{mm}$ and the measurement domain extends as sketched in Figure 5.

\section{Tomographic reconstruction}

The volumetric light intensity reconstruction is performed following the of multiplicative algebraic reconstruction technique (MART) ${ }^{57}$ algorithm by Lavision software Davis 7.4. A three-dimensional mapping function from image-space to physical object-space is generated by imaging a calibration target. The initial experimental errors due to system calibration are estimated at approximately 0.5 pixels by the disparity vector field. ${ }^{58}$ The misalignment is reduced to less than 0.05 pixels making use of the self-calibration technique..$^{59}$ The raw images are pre-processed with subtraction of the minimum intensity at each pixel for the entire sequence, followed by a subtraction of the local minimum over a kernel of $31 \times 31$ pixels. The MART algorithm is applied with four iterations with a unit value of the pixel-to-voxel ratio.

In the circular jet, the illuminated volume of approximately $30 \mathrm{~mm}$ diameter and $50 \mathrm{~mm}$ height is discretized with $600 \times 600 \times 1000$ voxels. In the chevron case, instead it is $40 \mathrm{~mm}$ height and it is discretized by $600 \times 600 \times 750$ voxels. In both configurations, the resulting digital resolution is 20 voxels $/ \mathrm{mm}$ (voxel pitch of $50 \mu \mathrm{m}$ ). The accuracy of the reconstruction is evaluated a-posteriori comparing the intensity of reconstructed particles in the illuminated region with
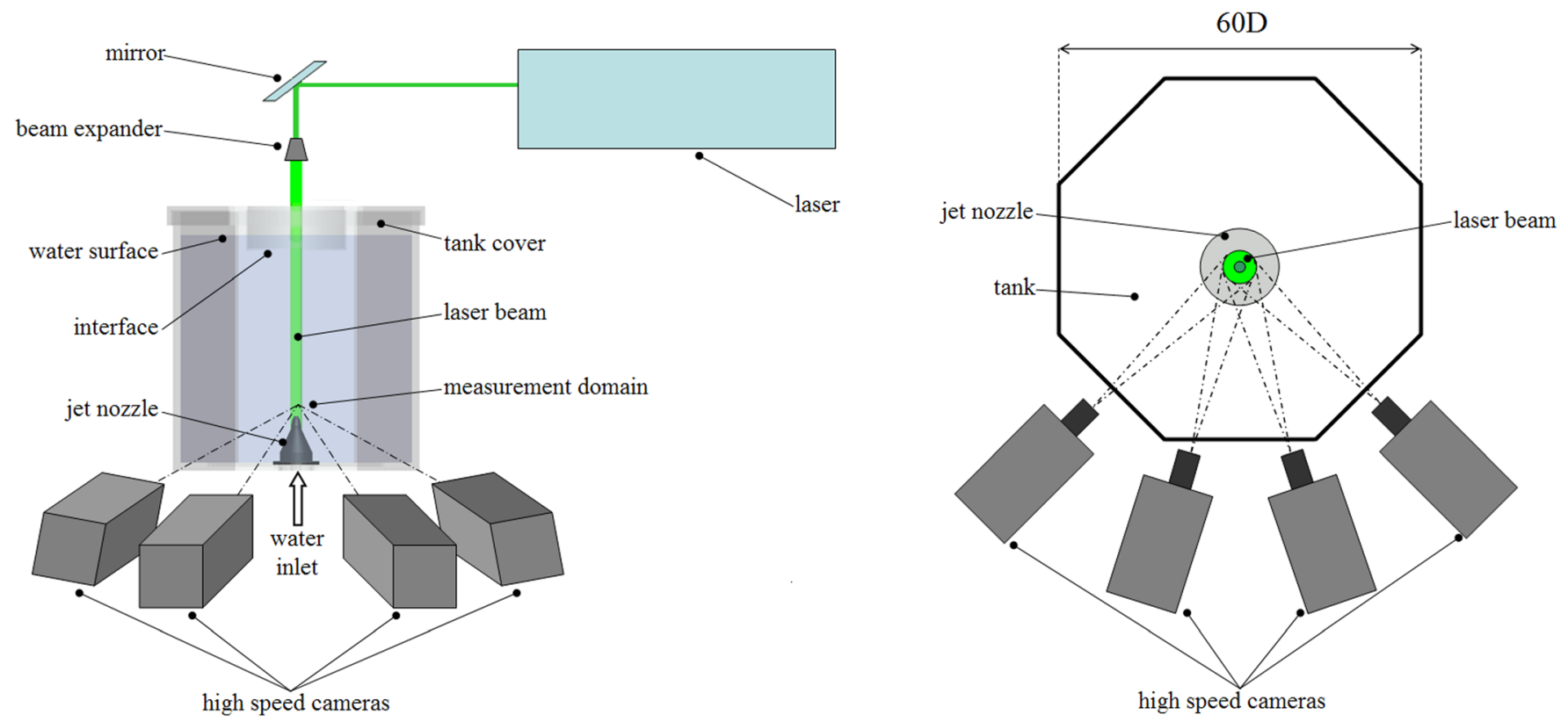

FIG. 3. (Color online) Schematic view of illumination and imaging in the tomographic experiment (left); top view of the system (right). 
TABLE I. Experimental parameters.

\begin{tabular}{|c|c|c|c|}
\hline & & Planar PIV & TOMO PIV \\
\hline \multirow[t]{2}{*}{ Seeding } & Diameter $[\mu \mathrm{m}]$ & 10 & 56 \\
\hline & Concentration [particles $/ \mathrm{mm}^{3}$ ] & 6 & 0.65 \\
\hline Illumination & $\begin{array}{l}\text { Quantronix Darwin-Duo Nd-YLF laser } \\
(2 \times 25 \mathrm{~mJ} @ 1 \mathrm{kHz})\end{array}$ & Sheet thickness $1.5 \mathrm{~mm}$ & Beam diameter $30 \mathrm{~mm}$ \\
\hline Recording device & $\begin{array}{l}\text { Photron fast CAM SA1 cameras } \\
(1024 \times 1024 \text { pixels @ } 5.4 \mathrm{kHz}) \\
20 \mu \mathrm{m} \text { pixel pitch }\end{array}$ & 2 cameras & 4 cameras \\
\hline Optical arrangement & $\begin{array}{l}\text { Nikon objectives (f; f\#) } \\
\text { field of view }\end{array}$ & $\begin{array}{l}105 \mathrm{~mm} ; 2.8 \\
4.7 D \times 9.2 D\end{array}$ & $\begin{array}{l}105 \mathrm{~mm} ; 32 \\
5 D \times 5 D\end{array}$ \\
\hline Acquisition frequency & & $1.2 \mathrm{kHz}$ & $1 \mathrm{kHz}$ \\
\hline Pulse separation & & $1 / 1200 \mathrm{~s}$ & $1 / 1000 \mathrm{~s}$ \\
\hline Number of recorded images & & 4000 & 500 \\
\hline
\end{tabular}

that of ghost particles produced in the immediate surrounding of the illuminated domain. ${ }^{19}$ A signal-to-noise ratio may be defined as the reconstructed particles intensity inside the illuminated area versus that reconstructed outside. In the present experiment, the intensity distribution of the laser light is approximately Gaussian and the light is concentrated in a circular region of approximately 550 voxels of diameter (Figure 6), corresponding to $27.5 \mathrm{~mm}$. The peak intensity profile is extracted along the radial direction $r$ shows that the intensity outside of the illuminate region is fairly negligible, which indicates a high signal-to-noise ratio.

\section{Vector field computation}

The three-dimensional particle field motion is computed by volume deformation iterative multigrid (VODIM) technique $^{51}$ with a final interrogation volume of $40 \times 40 \times 40$ voxels $\left(2 \times 2 \times 2 \mathrm{~mm}^{3}\right)$ with an overlap between adjacent interrogation boxes of $75 \%$, leading to a vector pitch of $0.5 \mathrm{~mm}$. At the given particle concentration, 6.5 particles are counted, on average, within the interrogation box.

A further refinement of the analysis is obtained by using a correlation averaging technique whereby the cross-correlation map, obtained over three subsequent object-pairs, is averaged yielding higher signal-to-noise ratio and higher measurement precision. Such approach is an extension of the method proposed by Meinhart et al. ${ }^{60}$ that is here extended to unsteady velocity fields, when the sampling rate exceeds that strictly needed for temporally resolving the flow. The use of such averaging technique involves a longer measurement time interval corresponding to the separation of four exposures $(3 \mathrm{~ms})$.

Data processing is performed on a dual quad-core Intel Xeon processor at $2.83 \mathrm{GHz}$ with $8 \mathrm{~GB}$ RAM memory

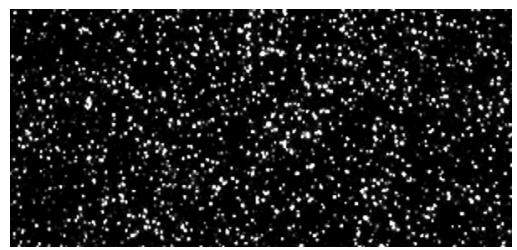

FIG. 4. Sample of $(300 \times 143$ pixels) particle image recording (after subtraction of the local minimum over a $31 \times 31$ pixels kernel). requiring, respectively, $20 \mathrm{~min}$ and $1 \mathrm{~h}$ for the reconstruction of a pair of objects and 3D cross-correlation.

\section{Time-resolved planar measurements}

Neutrally buoyant polyamide particles of $10 \mu \mathrm{m}$ diameter are employed with a concentration of approximately 6 particles $/ \mathrm{mm}^{3}$. The illumination and imaging is provided by the same equipment as used for the tomographic experiments. A laser-light sheet of $1.5 \mathrm{~mm}$ is formed applying a knife-edge slit-filter to the expanded beam. Two cameras are combined to cover a measurement region extending from the exit up to 10 diameters. The cameras are equipped with objectives of $105 \mathrm{~mm}$ focal length set at numerical aperture $f_{\#}=2.8$ to maximize light intensity. To avoid the phenomenon of peak-locking, ${ }^{61}$ the focal plane is slightly offset from the illumination plane (defocussing) and the particle images are blurred over an area of approximately $2 \times 2$ pixels. Sequences of images of tracer particles are recorded at frequency of $1.2 \mathrm{kHz}$ yielding a particle image displacement of approximately 7 pixels at the jet exit.

The field of view (FOV) of each camera is $47 \mathrm{~mm} \times 47 \mathrm{~mm}$, with a digital resolution of 20.7 pixels $/ \mathrm{mm}$. The two FOVs overlap of $2 \mathrm{~mm}$ and, when combined, form a measurement domain of $47 \mathrm{~mm} \times 92 \mathrm{~mm}$. The experimental settings are summarized in Table I.

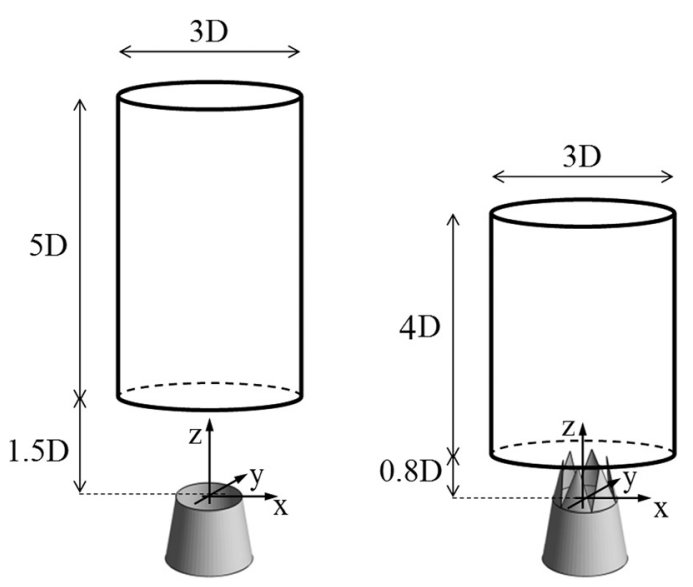

FIG. 5. Details of the measurement and coordinate system. 


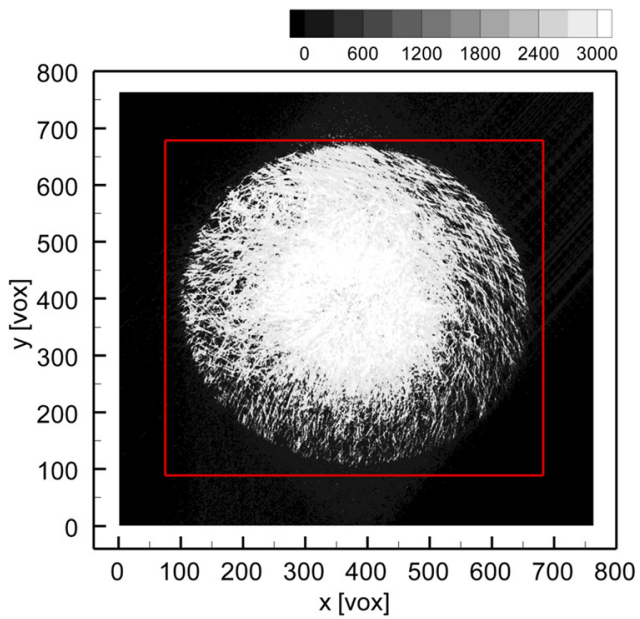

The time-averaged velocity profile at the exit, where the flow regime is steady and laminar, is computed by ensembleaveraging the correlation signal along the measurement sequence. ${ }^{60}$ As a result, the interrogation window can be decreased down to $6 \times 6$ pixels $\left(0.29 \times 0.29 \mathrm{~mm}^{2}\right)$ with an overlap factor of $50 \%$ leading to a vector pitch of $0.13 \mathrm{~mm}$.

\section{Uncertainty analysis for tomographic PIV}

A reliable estimate of the measurement uncertainty of three-dimensional time resolved measurements can be performed by several a posteriori approaches based on physical criteria. ${ }^{51,62}$ At the present measurement rate, temporal fluctuations are sampled by a large number of snapshots, where the measurement error is expected to be uncorrelated. The measurement precision error can be evaluated by comparing the velocity time history to a filtered version obtained with a kernel length smaller than the flow scales. Such a comparison is shown in Figure 7, in which the raw and the filtered data are plotted at a given point in the shear layer $(x=0.5 D, y=0, z=3 D)$, where the flow unsteady behavior is dominated by the passage of laminar vortex rings. Each velocity component is filtered with a second order polynomial least squares regression ${ }^{63}$ over a kernel of size $L$ in space and duration $T$. In order to avoid significant amplitude modulation, which would mean a distortion of large-scale flow structure characteristics, the kernel size must satisfy the following relations: $L<d_{V}$ and $T<d_{V} / W_{V}$, where $d_{V}$ is the core diameter of a vortex moving with a velocity $W_{V}$. An estimate of the characteristic kernel size, for instance, can be based on laminar vortex rings
FIG. 6. (Color online) (Left) Reconstructed particle peak intensity distribution averaged along $z$-axis (the red box indicates the reconstructed region); (right) normalized particle peak intensity profile along the radial direction (azimuthal average).
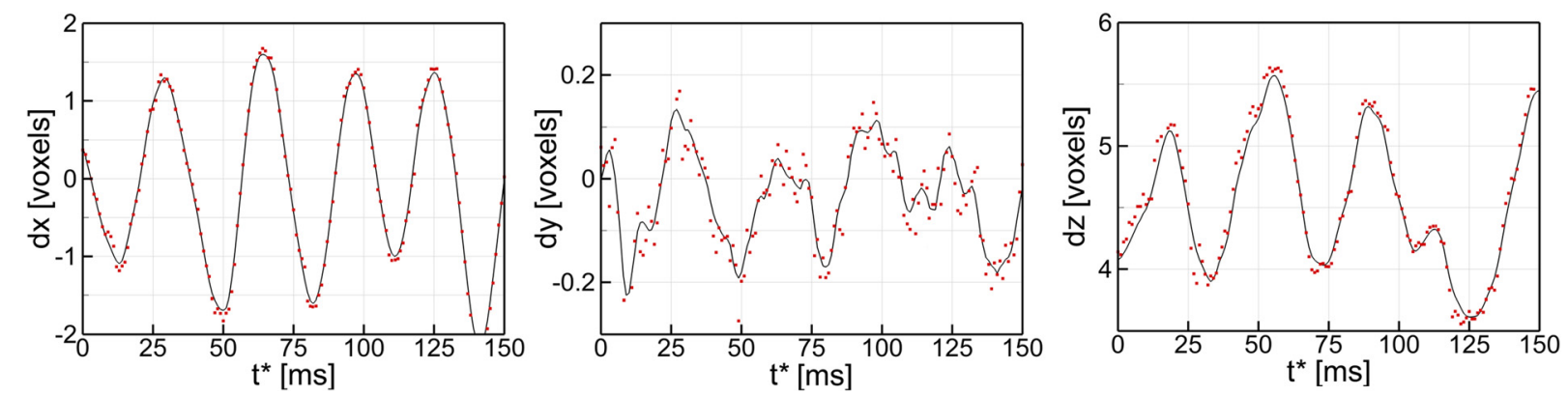

FIG. 7. (Color online) Time history of the velocity components at ( $x=0.5 D, y=0, z=3 D)$. Raw data (red dots) and second order time-space filtered data (solid black line). 


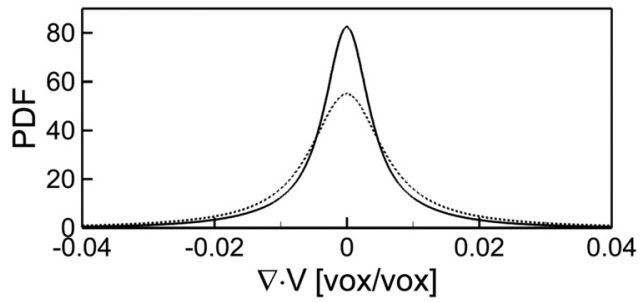

FIG. 8. Probability density of measurement error estimated by velocity divergence. Raw data (dotted line) and filtered (solid line).

must be satisfied at all time instants. The numerical evaluation of $\nabla \cdot \mathbf{V}$ from experimental data may differ from zero due to measurement error on the velocity and the numerical truncation in the spatial discretization. As discussed in Ref. 51, the relatively large overlap factor between neighboring interrogation boxes ensures that truncation errors are negligible with respect to the finite size of the interrogation. Hence, the standard deviation of the velocity divergence over the entire measurement domain and during the whole observation time $\langle|\nabla \cdot \mathbf{V}|\rangle$ can be used as statistical estimate of the measurement precision for the velocity gradient tensor components. Figure 8 illustrates the probability density function of $\nabla \cdot \mathbf{V}$. The dotted and solid lines correspond to the raw and the filtered data. The error shows a Gaussian distribution symmetrical around zero with a standard deviation $\langle|\nabla \cdot \mathbf{V}|\rangle$ of 0.012 and 0.007 voxels/voxel for the raw and the filtered data, respectively. The measurement of the terms on the trace of the velocity gradient tensor $\frac{\partial u}{\partial x}, \frac{\partial v}{\partial y}$, and $\frac{\partial w}{\partial z}$ is usually less accurate than that of the non-diagonal terms; therefore, the above values may be considered as conservative estimates for the uncertainty of all the velocity gradient components. Considering the particular case of the vorticity, dividing the absolute error by the typical value measured in the core of the shed vortex ( 0.25 voxels/voxel), the resulting relative precision error on the vorticity field is estimated to be less than $3 \%$.

In the region where the fluctuations are dominated by laminar vortex rings, the vorticity residual evaluated as the difference between the filtered and raw data exhibits a standard deviation of 0.01 voxels/voxel, which is comparable to the above estimate of the measurement uncertainty.

\section{RESULTS}

\section{A. Statistical flow properties}

The mean velocity profile of the circular jet at the nozzle exit was obtained through the sum of correlation of PIV images leading to a 77 vectors along the diameter (Figure 9). The profile is taken $1 \mathrm{~mm}$ downstream of the exit to avoid using data corrupted by laser light reflections from the nozzle surface. The shear layer has a normalized displacement $\delta_{e} / D$ and momentum thickness $\theta_{e} / D$ of 0.078 and 0.028 , respectively. These are both evaluated between the radial positions where the mean axial velocity is $0.1 W_{j}$ and $0.9 W_{j}$, where $W_{j}$ is the maximum axial velocity of the extracted profile (Figure 9). The ratio $\delta_{e} / \theta_{e}=2.78$ agrees well with the theoretical value for the Blasius solution of $2.59 .^{24}$ Moreover, $\theta_{e} / D$ satisfies the relation for laminar shear layers

$$
\theta_{e} / D=c\left(\frac{W_{j} D}{\nu}\right)^{-0.5},
$$

with $c=1.2 .^{25}$ The level of axial velocity fluctuation $w^{\prime}$ in the jet core is less than $0.5 \% .^{12,24,65}$

The physical quantities presented in the rest of the paper are reported in non-dimensional units dividing by the jet diameter $D$ or exit velocity $W_{j}$. The normalized time is $t=t^{*} W_{j} / D$ and the normalized spatial coordinates are $X=x / D, Y=y / D$, $Z=z / D, R=r / D$; the corresponding normalized velocity components are $U=U^{*} / W_{j}, V=V^{*} / W_{j}, W=W^{*} / W_{\mathrm{j}}, V_{r}=V_{r}^{*} / W_{j}$ and, similarly, the normalized velocity fluctuations are $u^{\prime}=u^{* \prime} / W_{j}, v^{\prime}=v^{* \prime} / W_{j}, w^{\prime}=w^{*^{\prime}} / W_{j}, v_{r}^{\prime}=v_{r}{ }^{\prime} / W_{j}$.

The spatial development of the jet is illustrated by contours of mean axial velocity (Figure 9) and on several crosssectional planes for the chevron configuration (Figure 10). In both configurations, $95 \%$ of the axial velocity is still maintained beyond $Z=4,3,36,37$ and in the circular case, where the measurements are available till 7.5 diameters, it is conserved up to $6.5{ }^{9,66}$ Compared to the axisymmetric development of the circular case, the shear layer of the chevron is shaped from the nozzle exit with a star-like pattern as a result of the chevron geometry. ${ }^{3,36,37}$ Most evident between $Z=1$ and 2, a secondary flow pattern of higher axial velocity is installed in correspondence to the notch where the radial outflow $\left(\approx 0.1 W_{j}\right)$ rolls-up into a circulatory motion slightly staggered with respect to the chevron apex. The secondary flow
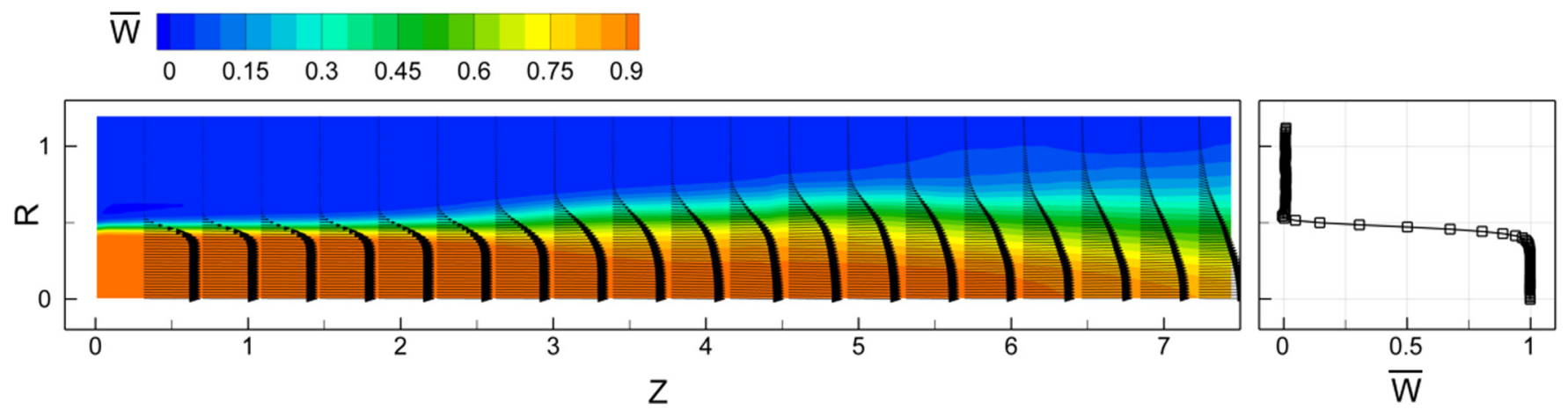

FIG. 9. (Color online) Circular jet: iso-contours of mean-axial velocity (left) and jet: cross-sectional profile of mean-axial velocity at $\mathrm{Z}=0.1$ (right). 

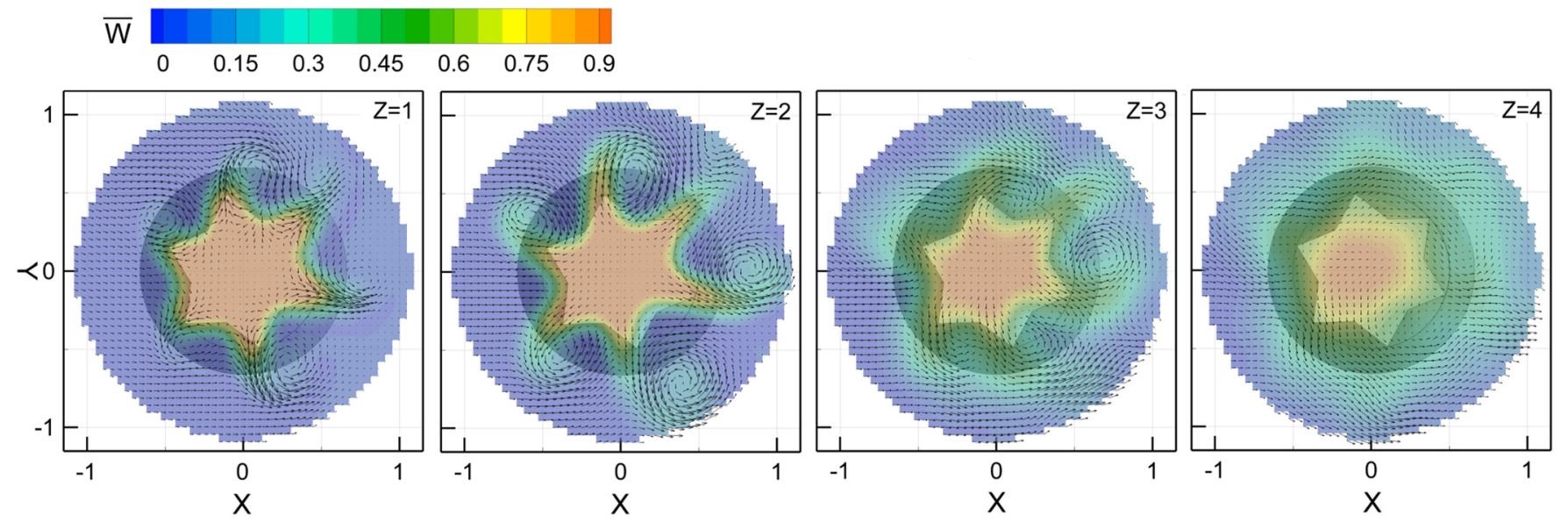

FIG. 10. (Color online) Chevron jet: contours of mean-axial velocity at different cross-sectional Z-planes; X-Y projection of velocity vectors.

develops either on the left or the right side of the valley and does not occur with fully regular organization, which is ascribed to small imperfections of the boundary conditions. The investigations by Bridges and Brown, ${ }^{3}$ Opalski et al., ${ }^{36}$ and Alkislar et al. ${ }^{37}$ were conducted at higher values of the Reynolds number and showed lobed patterns centered at the chevron notch, which suggests the tendency of secondary rollers to be aligned with the notch as Re increases. On the other hand, similarly to what reported by Opalski et al. ${ }^{36}$ and Alkislar et al. ${ }^{37}$ the flow loses the characteristic lobed pattern and gradually gains axisymmetric coherence between $Z=3$ and 4 .

To observe the effect of chevrons on the development of the shear layer, mean axial velocity profiles corresponding to the chevron apex and notch (Figure 11) are compared to the circular case in the radial direction (Figure 12). The velocity profile at the notch and apex are obtained by averaging the velocity from the six azimuthal locations. The shear layer thickness $\delta$ is defined as the distance between the radial locations where $W=0.1$ and 0.9 , at $Z=1$. The profile extracted at the notch is characterized by a thicker shear layer $(\delta=0.28)$ compared to that at the apex $(\delta=0.14)$ and the circular case $(\delta=0.17)$. In fact, the chevrons induce a stretching of the shear layer ${ }^{37}$ with a $15 \%$ smaller thickness at the apex and an increase up to $50 \%$ at the notch, when compared to the circular case. More downstream, at $Z=3$, the time-averaged profiles of axial velocity show a much closer trend. At the apex, the shear layer thickness $\delta(0.3)$ becomes $10 \%$ smaller than in the circular case, while it is $17 \%$ larger at the notch.

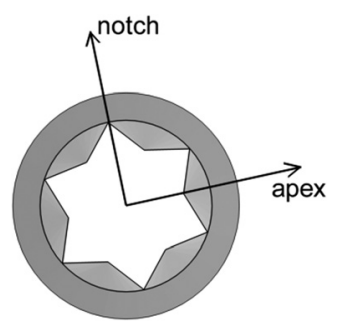

FIG. 11. Azimuthal position of chevron notch and apex.
The turbulent fluctuations also exhibit marked differences between the circular and the chevron jet exit. In Figure 13 , axial and radial velocity fluctuations, respectively $w^{\prime}$ and $\mathrm{v}_{\mathrm{r}}^{\prime}$, are compared along the jet axis. In the circular jet, the axial fluctuation increases rapidly, reaching a peak of 0.17 at $Z=3.2$. For a circular jet at $R e=12000$, Zaman and Hussain $^{24}$ associated the peak activity $\mathrm{w}^{\prime}=0.11$ at $Z=3$ to the pairing of vortex ring structures. At higher Reynolds $(R e=55000)$, the same authors ${ }^{66}$ found $w^{\prime}>0.15$ between $Z=2.5$ and 7 and observed that, when the jet was acoustically excited at the vortex pairing frequency, the axial fluctuation showed again a peak at the pairing position $(Z=3)$. Also Ganapathisubramani et al. ${ }^{13}$ reported peak values of axial velocity fluctuations associated to the pairing phenomenon. Further downstream $\left(Z>4\right.$, Figure 13), $w^{\prime}$ decreases to 0.11 . Similar observation was reported by Yule ${ }^{27}$ who found that all the fluctuating velocity components of jet flows ranging between $R e=9000$ and $2 \times 10^{5}$ tend to a constant value of 0.15 .

In the chevron case, the axial velocity fluctuation increases to 0.07 in the first three jet diameters and then it remains nearly constant, which means that, compared to the circular case, at $R e=5000$, chevrons are responsible for a considerable reduction of axial fluctuations (up to $40 \%$ ). For a compressible chevron jet at $R e=1.3 \times 10^{6}$, Alkislar et al. ${ }^{37}$ reported the tendency of the peak value of $\mathrm{w}^{\prime}$ to an asymptotic value of 0.12 , which, for $Z>3$, was $25 \%$ smaller than that of the circular jet.

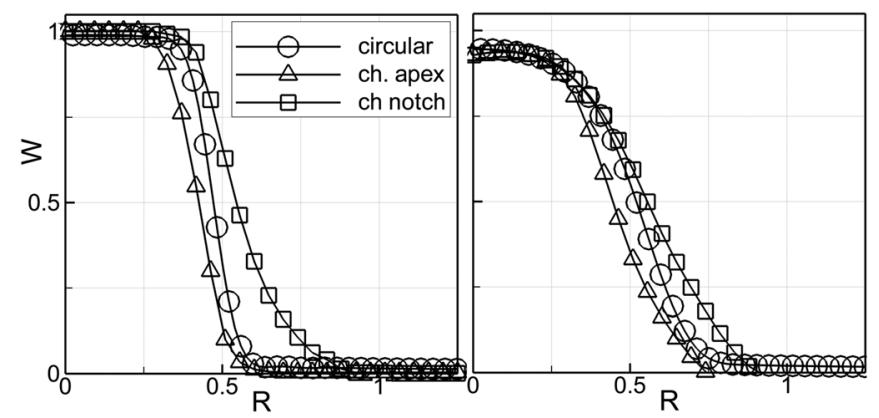

FIG. 12. Mean axial velocity profiles at $Z=1$ (left) and $Z=3$ (right). 


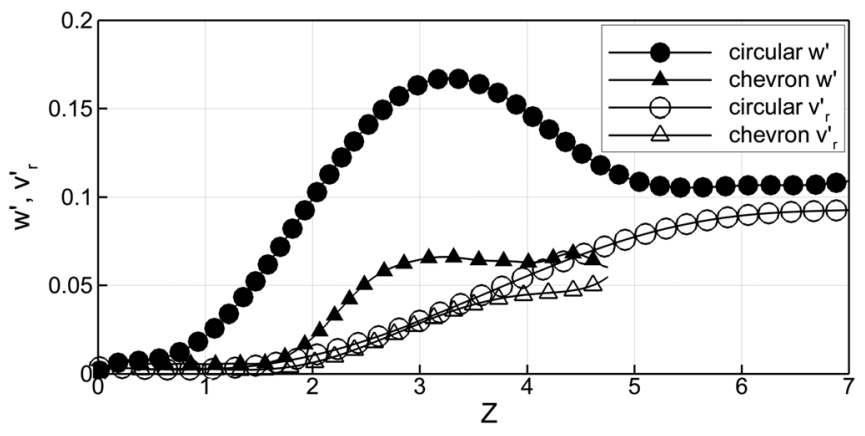

FIG. 13. Axial and radial velocity fluctuations along the jet axis.

In the circular jet, the radial turbulence intensity $v^{\prime}{ }_{r}$ gradually increases along the jet axis reaching a nearly constant value of 0.09 between $Z=6$ and 7, which is in agreement with experiments at higher Reynolds number by Yule, ${ }^{27}$ Hussain and Zaman, ${ }^{66}$ and Alkislar et al. ${ }^{37}$ In the chevron configuration, $v_{r}^{\prime}$ increases with the same rate of the circular jet until $Z=3.5$ and then levels out to 0.05 meaning of a radial turbulence suppression up to $25 \%$.

Cross-sectional profiles of $w^{\prime}, v_{r}^{\prime}$ and transverse Reynolds stress $w^{\prime} v^{\prime}{ }_{r}$ of the circular and chevron jet are compared in Figure 14. At $Z=1$, the turbulent fluctuations peak along the position of the shear layer. Axial velocity fluctuation $w^{\prime}$ is approximately 50\% higher in the chevron nozzle than the circular, with peaks of 0.11 , indicating a more rapid onset of the turbulent regime. In contrast, the radial fluctuation $v_{r}^{\prime}$ is higher in the circular jet with peak value of 0.08 , which is 8 times smaller at the chevron apex and 3 times at the notch. The presence of axisymmetric vortex structures causes radial fluctuations of amplitude comparable to those in the axial direction. At the notch axial and radial turbulence reach peaks of 0.1 and 0.03 , respectively, at radial coordinate $R=0.45$. At the apex, peak values are observed at $R=0.35$, which also correspond to the radial position of maximum $w^{\prime}$ and $v_{r}^{\prime}$ in the circular jet. In the chevron jet, the transverse Reynolds stress $w^{\prime} v_{r}^{\prime}$ at the apex is one order of magnitude smaller than the peak activity at the notch (0.0033). Maximum of $w^{\prime} v^{\prime}{ }_{r}=0.0012$ is observed for the circular configuration.

An overall increase of the turbulent fluctuations is observed at $Z=3$ for both configurations. In the circular jet, axial turbulence shows a maximum $(0.21)$ at $R=0.25,50 \%$ larger than in the chevron case. Profiles of $w^{\prime}$ along the apex and the notch directions show a similar trend reaching 0.1 at $R=0.25$. Further away from the axis, the apex profile drops to 0.03 at $R=0.6$, whereas the notch profile reaches a peak $(0.11)$ on the side of lower speed $(R=0.4)$ and gradually
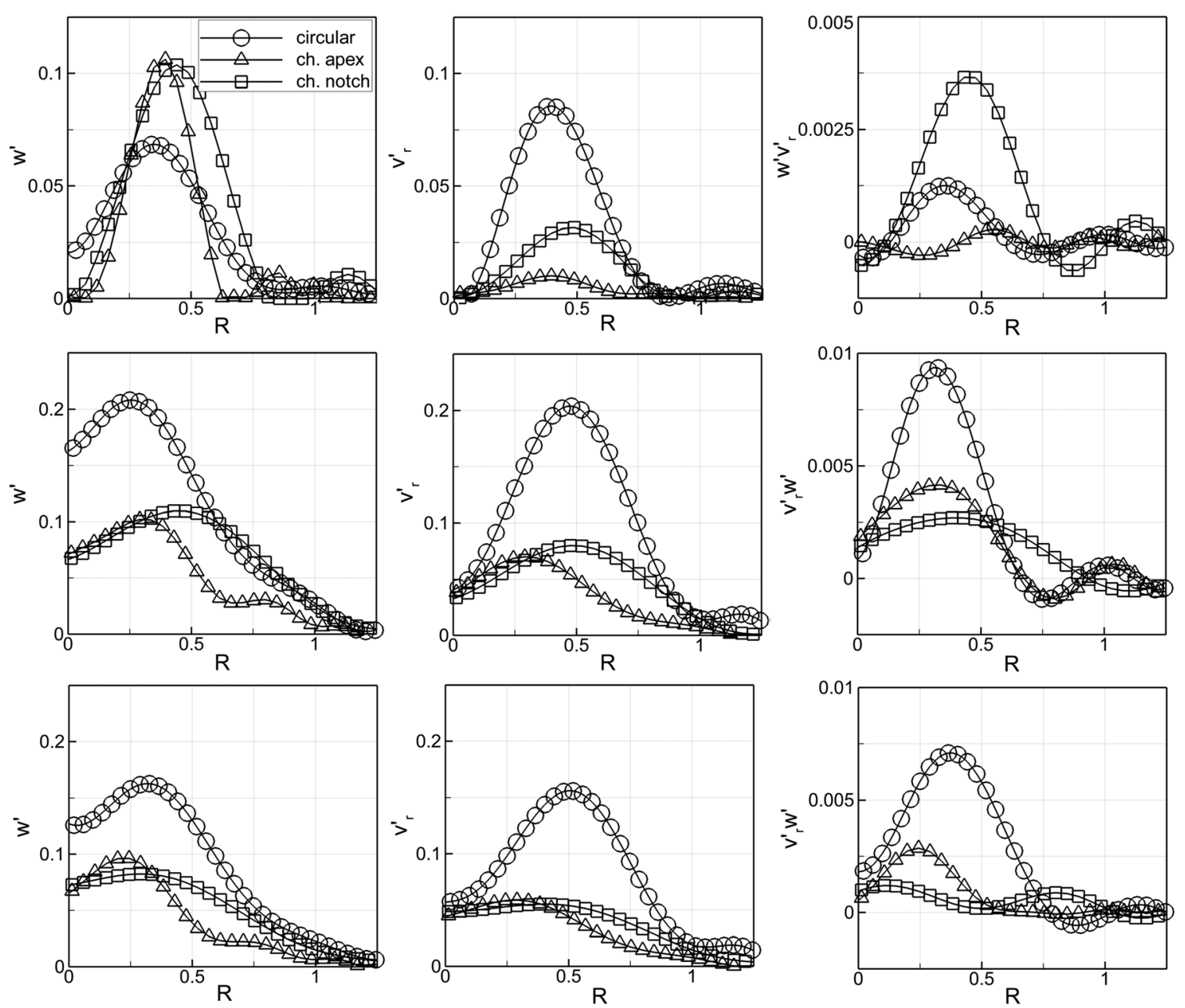

FIG. 14. Axial and radial velocity fluctuation and transverse Reynolds stress at cross section $Z=1$ (top), $Z=3$ (centre), $Z=4.5$ (bottom). 
decreases with a rate comparable to that of the circular case. For the radial velocity fluctuations $v_{r}^{\prime}$, the circular configuration shows peak activity $(0.2$ at $R=0.5)$ of intensity comparable to the axial peak turbulence. In the chevron case, the maximum values of $v_{r}^{\prime}$ correspond to those of $w^{\prime}$, although reduced by $60 \%$ with respect to peak activity found in the circular jet. Peaks in axial and radial turbulent intensity are associated with the vortex pairing process. ${ }^{22}$ The chevron apex profile shows a peak $w^{\prime} v^{\prime}{ }_{r}=0.004$ in the region of higher velocity $(R=0.3)$ that is $40 \%$ larger than that in the notch direction $\left(w^{\prime} v^{\prime}{ }_{r}\right.$ at $\left.R=0.4\right)$. However, when compared to the circular configuration, the apex and notch profiles feature maximum $50 \%$ and $70 \%$ smaller, respectively. Despite an overall increase of $30 \%$, profiles of $w^{\prime}, v_{r}^{\prime}$, and $w^{\prime} v^{\prime}{ }_{r}$ shown in this investigation are in agreement with Hussain and Zaman. ${ }^{66}$ On the other hand, no similarity is found with the cross-sectional profile at $Z=2$ shown in the investigation by Alkislar et al., ${ }^{37}$ which is ascribed to the considerable difference in Reynolds number.

At $Z=4.5$, axial and radial turbulence and Reynolds stress $w^{\prime} v_{r}^{\prime}$ of both configurations show a reduced peak activity when compared to what observed at $Z=3$. In the circular jet, turbulence peak activity is observed at $R=0.3$ for $w^{\prime}$ (0.17) and at $R=0.5$ for $v_{r}^{\prime}(0.16)$, corresponding to a $20 \%$ reduction compared to the absolute peaks at $Z=3$. A similar decrease is also found for the transverse Reynolds stress $w^{\prime} v^{\prime}{ }_{r}$. Bradshaw et al. ${ }^{22}$ reported a similar shear stress profile at $Z=4$ with peak activity of 0.009 at $R=0.5$. In the chevron configuration, peaks of $w^{\prime}$ reduces of $5 \%$ at the apex and $20 \%$ at the notch in comparison to the values at $Z=3$. Radial turbulence, instead, drops of $25 \%$ at the apex and $45 \%$ at notch. The Reynolds stress $w^{\prime} v_{r}{ }_{r}$ is attenuated too: at the apex, its peak decreases to 0.003 and is shifted towards the jet axis $(R=0.25)$, while $w^{\prime} v^{\prime}$ globally drops of approximately $50 \%$.

\section{B. Unsteady flow behavior}

\section{Axisymmetric regime of circular jet}

The most prominent unsteady feature exhibited by the circular jet flow is the shedding of axisymmetric vortices that result from the growth of Kelvin-Helmholtz instabilities. The shedding occurs regularly for a laminar jet exit as reported in previous works..$^{8-12}$ The instantaneous flow organization of the circular jet is illustrated in the temporal sequence of Figure 15 showing the Kelvin-Helmholtz vortices by iso-surfaces of normalized azimuthal vorticity $\omega_{\theta}=\omega_{\theta} * D / W_{j}$. The development towards the three-dimensional regime occurs mostly downstream of the pairing region and is visualized by the normalized axial and radial vorticity components $\omega_{z}=\omega_{z} * D / W_{j}$ and $\omega_{r}=\omega_{r} * D / W_{j}$, which are plotted separately. The visualization of the azimuthal, the radial, and the axial component of the vorticity vector enables an easier inspection of the flow organization, especially in the three-dimensional regime where streamwise structures develop together with azimuthal ones.

Figure 15 illustrates a temporal sequence of the flow field with snapshots separated by a normalized time $\Delta t=0.61$, which corresponds to approximately half the shedding period. The iso-surface of normalized vorticity $\omega_{\theta}=4$ identifies axisymmetric vortices which are labeled with " 1 ," " 2 ," "3," "4," and "5." The formation of vortex ring structures in the near region of circular jets is well known from several studies conducted over a wide range of Reynolds number from $10^{3}$ to $10^{5} \cdot 9,12,13,22,23,27$ In the early investigations, ${ }^{9,68}$ it was hypothesized that acoustical excitation at the natural shedding frequency or at the pairing frequency ${ }^{24}$ significantly stabilizes the temporal and spatial formation of the ring vortices, as well as the pairing. In later studies, this method enabled an accurate phase-locked analysis of the pairing mechanism. ${ }^{25}$

In the present experiment, the shedding is observed between $Z=2$ and 2.5, which agrees with the findings of Liepmann and Gharib ${ }^{12}$ and occurs at Strouhal number $(S t)$ of 0.72 , based on the jet exit velocity $W_{j}$ and diameter $D$, which also agrees with the investigation on a non-excited jet at $R e=5000$ by Becker and Massaro. ${ }^{8}$ The spacing between the vortex rings appears to be half of the nozzle diameter. The pattern of dilated and compressed streamlines induced by the ring vortices which are coplanar to the jet nozzle is often referred to as varicose mode. ${ }^{8}$ The core induces an outward flow at its leading edge and inward at the trailing one, which increases as the vortex ring grows and is convected downstream. At the leading edge of vortex rings, such as " 3 ," the radial velocity reaches peaks of 0.3 and the normalized vorticity $|\omega|$ at the core is 6 . This is shown in Figure 16 where the vortex rings are identified by iso-lines of $\lambda_{2}-$ criterion.

In the range between 2.7 and 3.5 diameters, where axial and radial fluctuations exhibit maximum amplitude $\left(w^{\prime}=0.2\right.$ and $v^{\prime}{ }_{r}=0.21$, Figure 14), the vortices undergo pairing ${ }^{9,42,66}$ with a frequency $S t=0.36$, half of that of shedding. The early study by Petersen ${ }^{65}$ on a circular jet at $R e=5000$ showed that that vortex pairing takes place at 3 jet diameters downstream the nozzle exit with a frequency of $S t=0.55$. With a tracking of the vortex cores in a $R e=5500$ jet, Liepmann and Gharib ${ }^{12}$ showed that the first pairing usually takes place before $Z=2.5$ jet diameters. For acoustically excited jets at low Reynolds number, the phenomenon was studied for different Strouhal numbers ranging from 0.1 to 2. Crow and Champagne ${ }^{9}$ reported that vortices pair can be stabilized at $S t=0.3$ for $R e=10500$ to 100000 , whereas Zaman and Hussain $^{24}$ and Schram $^{25}$ investigated cases with a stronger excitation of the so called "jet-column mode" at $S t=0.85$ $(R e=32000)$ and $0.93(R e=14000)$, respectively. Based on phase locked PIV measurements, Schram $^{25}$ found that, at $R e=14000$, vortex pairing, or leapfrogging, occurred at $Z \approx 1.8$.

The flow induced by the preceding vortex ("4") produces axial and inward accelerations in the jet core between vortex " 3 " and " 4 ." As a result, the pursuer vortex ("4") is stretched along the axis increasing in convective velocity to peaks of 0.49 and decreasing in diameter. On the other hand, due to the outward motion induced by follower ("4"), the preceding one ("3") stretches positively while widening in diameter and its convection speed decreases to 0.43 . As a consequence of the convective velocity difference, the distance between the two vortices gradually reduces and the pursuer vortex ("4") pairs with the preceding one (“3”). 

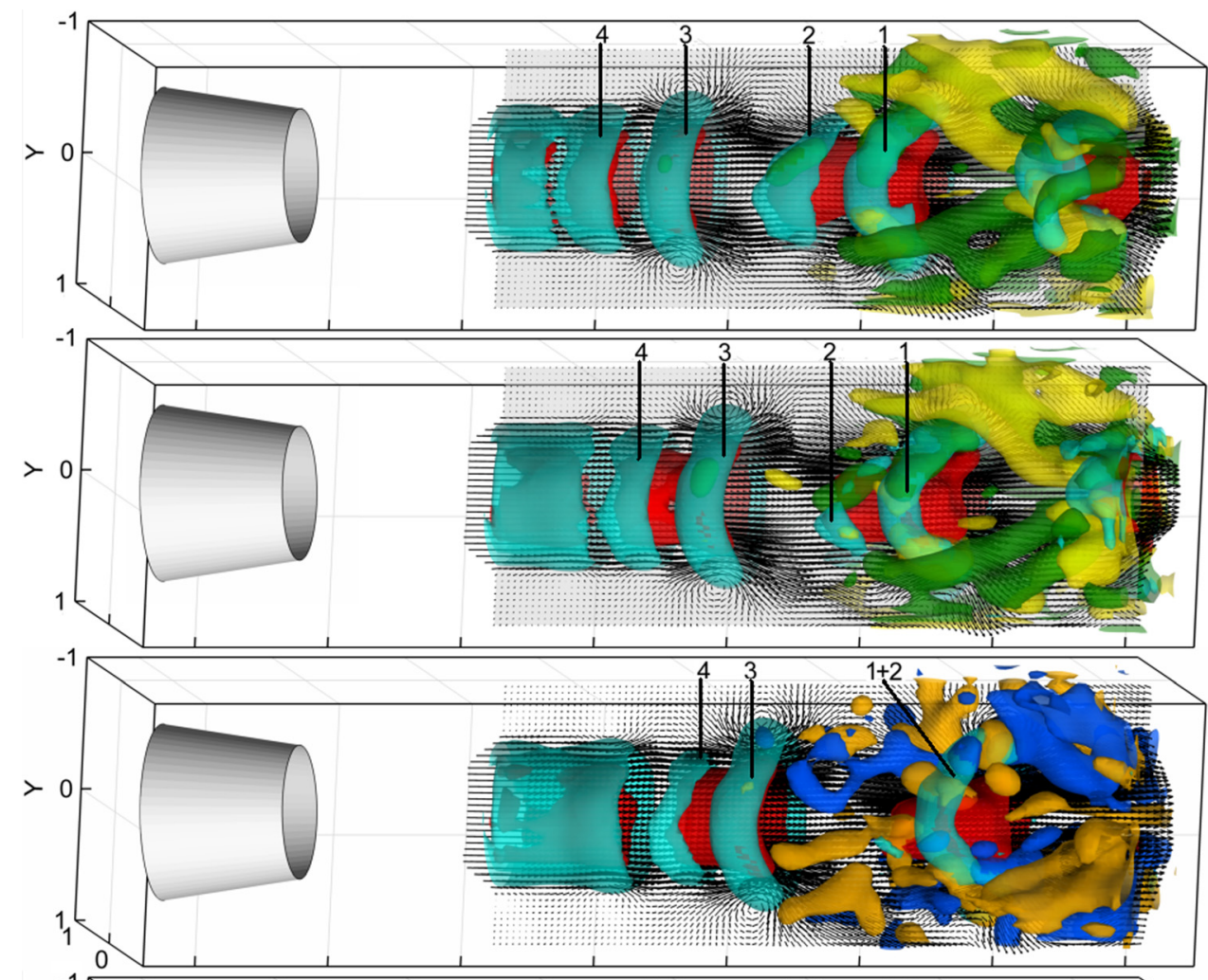

FIG. 15. (Color online) Time sequence visualization of the circular jet. Velocity vector slice in the axial plane. Isosurfaces of axial velocity $W=1.05$ (red). In the first two snapshots, iso-surface of vorticity components $\omega_{\theta}=4$ (cyan), $\omega_{z}=-1.2$ (green) and 1.2 (yellow); in the last two snapshots, iso-surfaces $\omega_{\theta}=4$ (cyan), $\omega_{r}=-1.2$ (blue) and 1.2 (orange). Time separation between displayed images $\Delta t=0.61$.

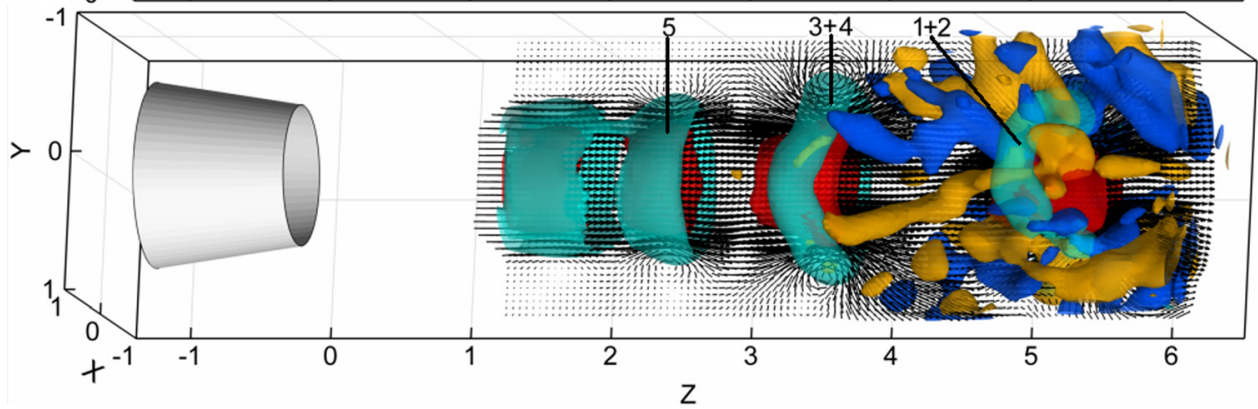

During pairing, as expected from angular momentum conservation, the peak vorticity at the core of trailing vortex reduces. For the trailing vortex, $|\omega|$ drops from 6 to 4 , while it increases to 7.5 at the leading one (Figure 16). At the end of the pairing, the resulting vortex (" $3+4$ ") has a convective velocity of 0.45 and induces a strong patch of flow at higher axial velocity of approximately 0.25 in the jet column. Axial velocity is maximum ( $W=1.15)$ within the vortex (" $3+4$ ") and it drops to $W=0.7$ in the upstream braid region. The induced outward motion, instead, gradually increases during pairing to absolute peak values of 0.35 at $Z=3$.

The convective velocity $W_{V}$ of vortex rings at $R e=14000$ was reported by $\mathrm{Schram},{ }^{25}$ who found $W_{V}=0.43$ for the preceding vortex, $W_{V}=0.64$ for the
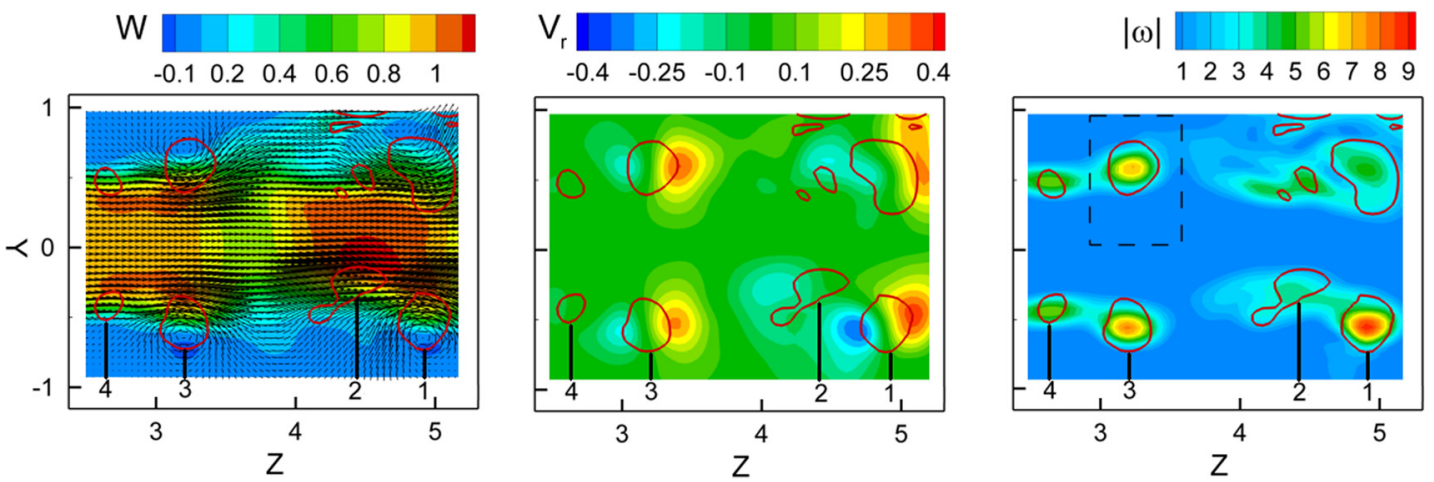

FIG. 16. (Color online) Iso-contour of axial and radial velocity (left and centre) and absolute vorticity | $\omega \mid$ (right) on plane $X=0$, iso-line of $\lambda_{2}=-0.5$, $t=0.61$. 
$\mathrm{t}=0.82$
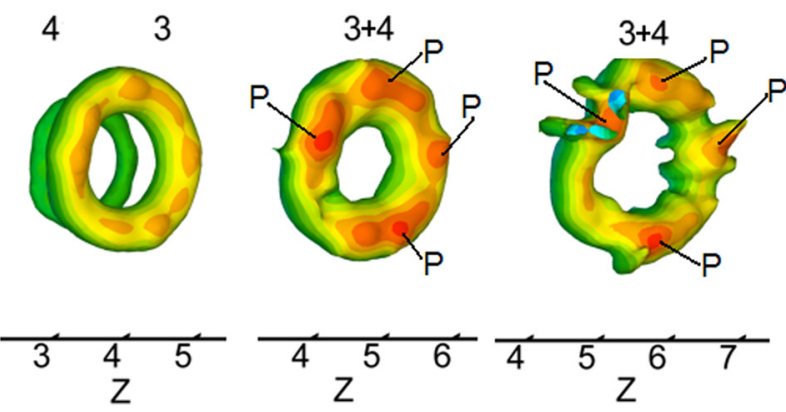

$\mathrm{t}=5.72$

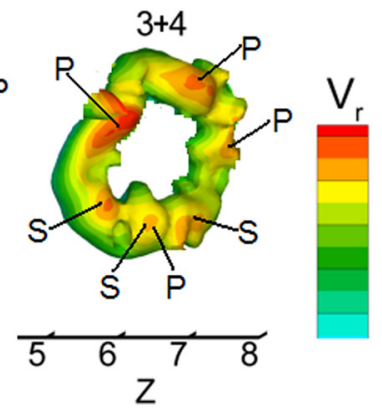

0.45

0.30

0.15

$-0.00$

$-0.15$
FIG. 17. (Color online) Time sequence visualization of vortex " $3+4$ " identified by iso-surface $\lambda_{2}=-0.5$ color coded with radial velocity component $V r$. Time separation between snapshots $\Delta t=1.64$. pursuer, and 0.61 for the merged. In this investigation, instead, vortices are detected by $\lambda_{2}$-criterion and convective velocities are evaluated at each time instant.

\section{Three-dimensional regime of circular jet}

During vortex pairing the flow pattern shows a significant growth of azimuthal instabilities. ${ }^{12,26,27}$ These may be further amplified during a second pairing event occurring downstream that leads to a further stretching of the distorted vortex. The growth of azimuthal modes of the ring vortices is accompanied by the formation of secondary vortices appearing just outside the region interested by the primary vortices. The secondary vortices develop in the streamwise direction. $^{12,13,22}$

Only few studies have applied volumetric approaches to investigate jet flow three-dimensional patterns, which still remain not fully understood. Hori and Sakakibara ${ }^{17}$ reported that, in the self-preserving region of a circular jet at $\operatorname{Re}=1000$, the jet interface is dominated by structures with elongated loop-vortices. Lynch and Thurow, ${ }^{15}$ by means of high speed three-dimensional flow visualization and proper orthogonal decomposition, showed azimuthal ring waves and streamwise instabilities in circular jets at $\mathrm{Re}=10000$. A quantitative discussion of the vorticity behavior at transition, which has not been yet undertaken, is given in the following paragraphs, together with the analysis of the corresponding stretching and tilting components (in Sec. III E).

After a vortex pairing event $(\mathrm{Z} \approx 3$, Figure 15$)$, the vortex rings gradually lose their axial symmetry, the azimuthal disturbances become more pronounced (see vortex " 1 " and " $1+2$ ") and counter-rotating streamwise filaments of radial and axial vorticity are formed. To understand the relation between the growth of azimuthal instabilities and the gradual loss of symmetry, the temporal evolution of vortex " $3+4$ " since its formation from vortex " 3 " and " 4 " is shown in Figure 17, where the iso-surface $\lambda_{2}=-0.5$ identifying the vortex is color coded with the radial velocity component $V_{r}$. When approaching the pairing $(t=0.82)$, although vortex " 3 " and " 4 " are axisymmetric, the radial velocity distribution on vortex " 3 " shows small irregularities with, respectively, peaks of 0.33 and -0.25 at the leading and trailing edge. After pairing $(Z=4.25, t=2.44)$, the axial symmetry of the vortex " $3+4$ " begins to decay and $V_{r}$ reaches 0.4 at the leading edge on four localized regions, which are labeled with "P." At $t=4.08$, these regions correspond to the peaks of azimuthal out-of-plane waves, whose amplitude doubles within $\Delta t=1.64$. At $\mathrm{t}=5.72$, three smaller azimuthal waves " $\mathrm{S}$ " deform the vortex ring shape and the flow motion in the azimuthal direction becomes comparable to $V_{r}$ (Figure 18). Comparatively, $\mathrm{Yule}^{27}$ reported the vortex rings gradually lose azimuthal coherence along the jet due to increasing circumferential velocity fluctuations. Vortex rings also undergo in-plane instability, which leads to the formation of 4 primary waves, which further rise between $t=3.28$ and 4.92 (in case of vortex " $3+4$ "), deforming the ring along two directions orthogonal to each other. This is illustrated in the temporal sequence of Figure 19 showing a top view of the vortex " $3+4$ " leading edge.

Early observations of vortex azimuthal instabilities were reported by Widnall and Sullivan ${ }^{26}$ who studied the vortex rings formed through acoustical excitation of an air jet and reported that the number of modes increases with $R e$. The same authors observed 6 in-plane modes at Reynolds number $(\Gamma / \nu)$ of 5790 , based on the circulation $\Gamma$ of the primary structures, and found that the number of waves increases with the Reynolds number. In the current experiments, which are conducted at Reynolds number $\Gamma / \nu=5000$ based on the circulation of the axisymmetric primary structures ( $\Gamma=\int_{S} \omega_{x} d S$ and surface $S$ defined by the dashed line of Figure 16), an average of 4 in-plane waves are observed.

The study of the dynamic flow properties associated with the large-scale structures must be based on data with time resolution satisfying, at least, the Nyquist criterion. With a $30 \mathrm{~Hz}$ cross-sectional PIV acquisition, Liepmann and Garib $^{12}$ showed the characteristic outward-inward radial

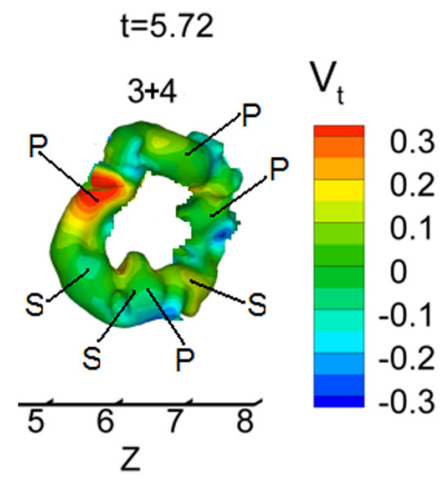

FIG. 18. (Color online) Iso-surface $\lambda_{2}=-0.5$ of vortex " $3+4$ " color coded with tangential velocity $V_{t}$. 
A-A view

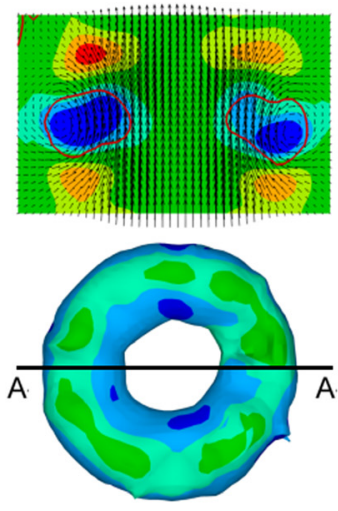

$t=1.455$
A-A view
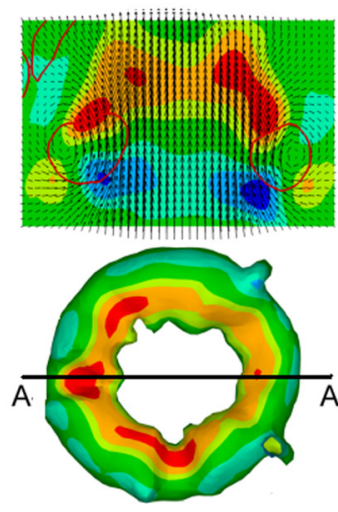

$t=1.94$

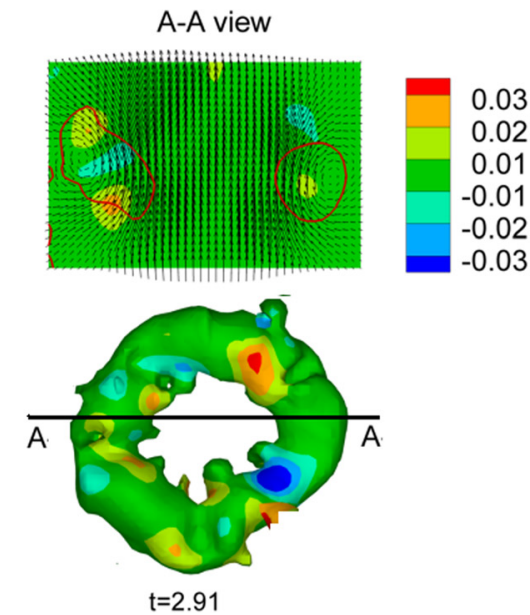

FIG. 19. (Color online) Visualization of vortex " $3+4$ " at three subsequent time instants. (Bottom) Top view of isosurface $\lambda_{2}=-0.5$ color coded with $d V_{r}$ l $d t$ (left), $d W / d t$ (centre), $d V_{t} / d t$ (right). (Top) Corresponding iso-contour on sectional plane A-A with iso-line $\lambda_{2}=-0.5$. motion induced by the passage of a vortex ring and evaluated the temporal evolution of the radial volume flux at different axial position. However, no information could be obtained on the related acceleration field, which is relevant to the decay of axisymmetric coherence of the vortex rings. Figure 19 illustrates the spatial distribution of the acceleration field associated with the vortex " $3+4$." On the bottom, vortex " $3+4$ " is shown at three subsequent time instants and is color-coded with the normalized axial and radial components of the acceleration $\left(d W / d t=\left(d W^{*} / d t^{*}\right) D / W_{\mathrm{j}}^{2} ; d V_{r} / d t=\left(d V^{*}{ }_{r} /\right.\right.$ $\left.d t^{*}\right) D / W_{j}^{2}$ ) which are also shown as contour plot on the sectional plane A-A. Between the instant of vortex pairing and that at which vortex " $3+4$ " travels outside of the measurement domain, the radial acceleration attains a maximum at $t=2.44$, corresponding to the first instant after the pairing. Despite being smaller than 0.01 on iso-surface $\lambda_{2}=-0.5$ and negative $(-0.03)$ in correspondence with the core, $d V_{r} / d t$ reaches values larger than 0.02 on the trailing and leading edge of the vortex cores, with asymmetric pattern. Maximum activity of 0.03 is observed at the leading edge of the left core (see corresponding contour plot). Further downstream, while $d V_{r} / d t$ shows an overall decrease at $t=3.28$, the axial acceleration reaches peaks of 0.03 on the leading side of the vortex and minima of comparable magnitude at the trailing edge, with asymmetric pattern along the azimuthal direction. Instead, peaks of normalized tangential acceleration $\left(d V_{t} / d t\right.$ $\left.=\left(d V_{t}^{*} / d t^{*}\right) D / W_{j}^{2}\right)$ are observed when the vortex is very deformed, such as at $t=4.92\left(\left|d V_{t} / d t\right|=0.03\right)$.

Between $Z=4$ and $Z=5$, a second pairing event is observed between vortex " 1 ," already resulting from a pairing, and vortex " 2 " ( $4<Z<5$, Figure 15). The instabilities grown during the first pairing appear to be further amplified by the second pairing, due to the larger axial and radial velocity fluctuations induced by the preceding vortex (" 1 "). In the present case, one observes a tilting up to $30^{\circ}$ of the trailing vortex ("2") while moving closer to vortex " 1 ." Temporal fluctuations at a half the frequency $(\mathrm{St}=0.16)$ are observed, which result in further amplification of the pulsating motion. The associated radial velocity reaches peaks of 0.38 and axial velocity shows instantaneous fluctuations ranging from 0.7 in the braid region $(3.5<Z<4)$ and 1.25 within " $1+2$ " $(4.25<Z<4.75)$ (Figure 16). The radial turbulence associated with the region of second pairing $(4<Z<5)$ increases to 0.08 along the jet axis (Figure 13), although its peak activity decreases to 0.15 , corresponding to a $25 \%$ drop compared to the first pairing $\left(Z=4.5\right.$, Figure 14). Here, the axial turbulence $\mathrm{w}^{\prime}$ reduces, without any relative maximum of along the jet axis (Figure 13), as it was instead observed for the first pairing. The reduction of the axial and radial turbulence accompanied by instantaneous fluctuation $w^{\prime}$ and $v_{\mathrm{r}}^{\prime}$ that are comparable or even larger than those found at the first pairing can be due to the lower temporal periodicity of second pairing. In addition, the flow threedimensionalities observed in this region tend to turn axial and radial fluctuations into the tangential ones. At $Z=5$, these are associated with tangential turbulence $v^{\prime}{ }_{\mathrm{t}}=0.12$.

Secondary flow instabilities are organized in counterrotating pairs of streamwise vortices, similarly to the streamwise structures of mixing layers, ${ }^{70}$ that contribute to both axial and radial vorticity given their inclination angle relative to the jet axis (iso-surface $\omega_{z}$ and $\omega_{r}$, Figure 15). The temporal sequence of Figure 20 illustrates the characteristic motion of the longitudinal filaments of $\omega_{z}=-1.2$ (green) and 1.2 (yellow) during the passage of vortex ring " $3+4$." At $Z=3.5(t=2.44)$, the streamwise structures are distributed around the vortex ring with an angle of approximately $30^{\circ}-40^{\circ}$ to the jet axis. ${ }^{22}$ The formation of streamwise instabilities is, however, observed further upstream, in the flow region dominated by the primary instabilities. ${ }^{12}$ In the braid region at $Z=2.5$, for instance, they reach peaks of absolute axial vorticity of 0.4 . Due to the flow motion induced by the ring vortex, streamwise structures move outward at the leading edge of the ring and inward at trailing edge, leading to a configuration where the filaments wrap the vortex ring $(t=4.92)$. The presence of longitudinal coherence inclined to the axis was reported in flow visualization investigations in circular jet flows at 2500 $<\operatorname{Re}<80000 .^{10,15,22,27}$ The early study by Bradshaw et $a l{ }^{22}$ suggested that the augmentation of streamwise coherence by artificial means, such as lobed nozzle or ejector shroud surrounding the nozzle, could be used to increase mixing properties and to reduce the acoustic emission. Yule $^{27}$ and Liepmann and Gharib ${ }^{12}$ observed that such structures develop in the braid region and accompany the pairing of the rings, and the latter authors added that the streamwise structures move in and out following the radial motion induced by the approaching vortex rings. 

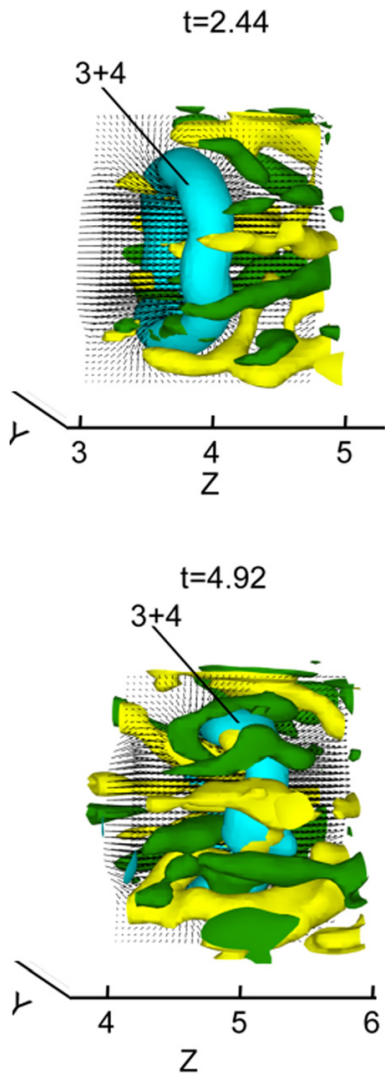

FIG. 20. (Color online) Time sequence visualization of iso-surface $\omega_{\theta}=4$ (cyan), $\omega_{z}=-1.2$ (green) and 1.2 (yellow).
The distribution and strength of the vortex filaments of $\omega_{z}$ at the leading and trailing edge of the vortex ring, as well as at $X-Y$ ring mid plane, are illustrated in Figure 21 showing crosssectional contours of axial vorticity of vortex " $3+4$ " at $t=2.44$ and $t=4.92$. At $t=2.44$, on the cross-section at the vortex ring trailing edge $(Z=3.2)$, a total of 11 streamwise instabilities $^{12}$ with axial vorticity magnitude $0.6<\left|\omega_{z}\right|<1.2$ occupy an approximately constant radial distance of 0.6 , and as shown by the velocity vectors, they are subjected to a rather uniform radial inward motion of 0.3 that is induced by the ring vortex. The difference in the number of waves between the azimuthal instability (4 primary waves) and the streamwise structure (11) suggests that these do not develop from the primary ring instabilities but are rather formed in the braid region. ${ }^{12}$ The cross-sectional plot at $Z=3.5$ illustrates the 4 longitudinal pairs ("STP") located in the vicinity of the external edge of the ring, with magnitude ranging from 0.5 to 1.5 . Due to the outward flow induced by the ring, the pairs are ejected towards larger radial distances $(R=0.8)$. At leading edge, the same 4 pairs of streamwise structures are immersed in the outward radial motion induced by the ring $\left(V_{r}=0.33\right)$. At $t=4.92$, the absolute axial vorticity of the streamwise structures increases up to 3 and remains uniform upstream $(Z=4.9)$ and downstream $(Z=5.5)$ the ring vortex. Due to the reduced azimuthal coherence of the flow, as it can be noticed comparing the velocity vectors at $t=4.92$ with $t=2.44$, the streamwise filaments no longer occupy a constant radial position and the visual identification of counter-rotating pairs becomes more difficult. The average number of longitudinal
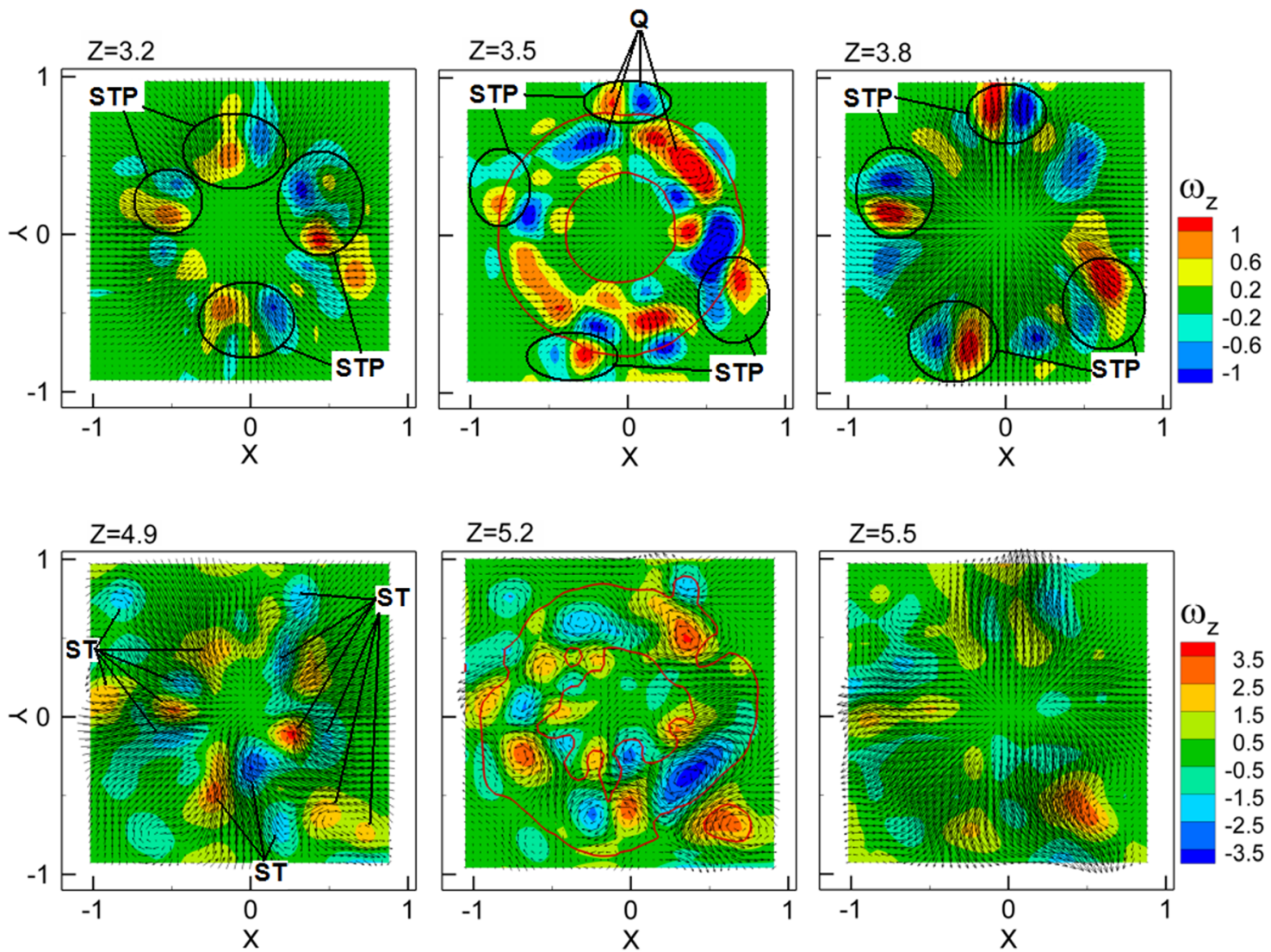

FIG. 21. (Color online) Cross-sectional iso-contours of axial vorticity $\omega_{z}$ at $t=2.44$ (top) and $t=4.92$ (bottom). $X$ - $Y$ projection of velocity vectors and with iso-line $\lambda_{2}=-0.5$. 

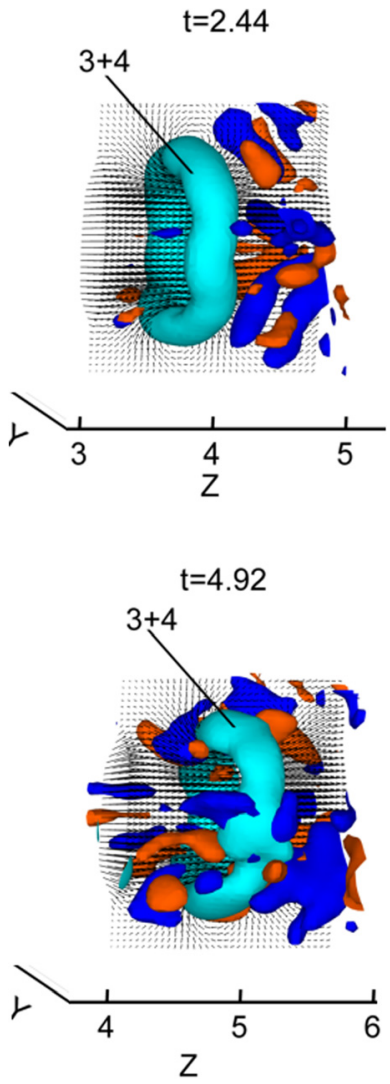

FIG. 22. (Color online) Time sequence visualization of iso-surface $\omega_{\theta}=4$ (cyan), $\omega_{r}=-1.2$ (blue) and 1.2 (red). structures ("ST") with a value of $\left|\omega_{z}\right|>1.5$ is 16 , which is larger in comparison to the upstream location $\mathrm{Z}=3.2$.

The latter observation does not seem to agree with the findings by Liepmann and Gharib ${ }^{12}$ who, based on laserinduced fluorescence (LIF) images, reported that the number of longitudinal structures decreases with the downstream distance. At $Z=5.2$, the number of axial swirl peaks associated with the primary structures is larger in comparison to what observed at $t=2.44$. On average, a total of 11 peaks with $\left|\omega_{z}\right|>2.5$ are found. In addition, compared to the cross sections $Z=4.9$ and 5.2, the activity of $\left|\omega_{z}\right|$ shows the tendency to drop at the vortex ring leading edge $(Z=5.5)$.

A detail of the streamwise radial coherence is illustrated in Figure 22 where two subsequent snapshots show that, similarly to "ST" vortices, the radial filaments "SR" move in the radial direction under the entrainment of vortex ring " $3+4$." At the leading edge of vortex " $3+4$," the filaments are distributed around the ring with an angle of approximately $30^{\circ}-$ $40^{\circ}$ to the jet axis $(Z=3.5, t=2.44)$. They are formed in the region of vortex rings, in conjunction with the growth of vortices "ST" $(Z=2.5)$, although showing peaks of radial vorticity $50 \%$ larger than those of the axial vorticity. The investigations by Yule $^{27}$ and Liepmann and Gharib ${ }^{12}$ reported the presence of streamwise vortices "ST," whereas they did not observe any streamwise coherence of radial vorticity, perhaps because the observations were only based upon cross-sectional measurements.

The organization and strength of the vortex filaments of $\omega_{r}$ ("SR") across " $3+4$ " are illustrated in Figure 23. At
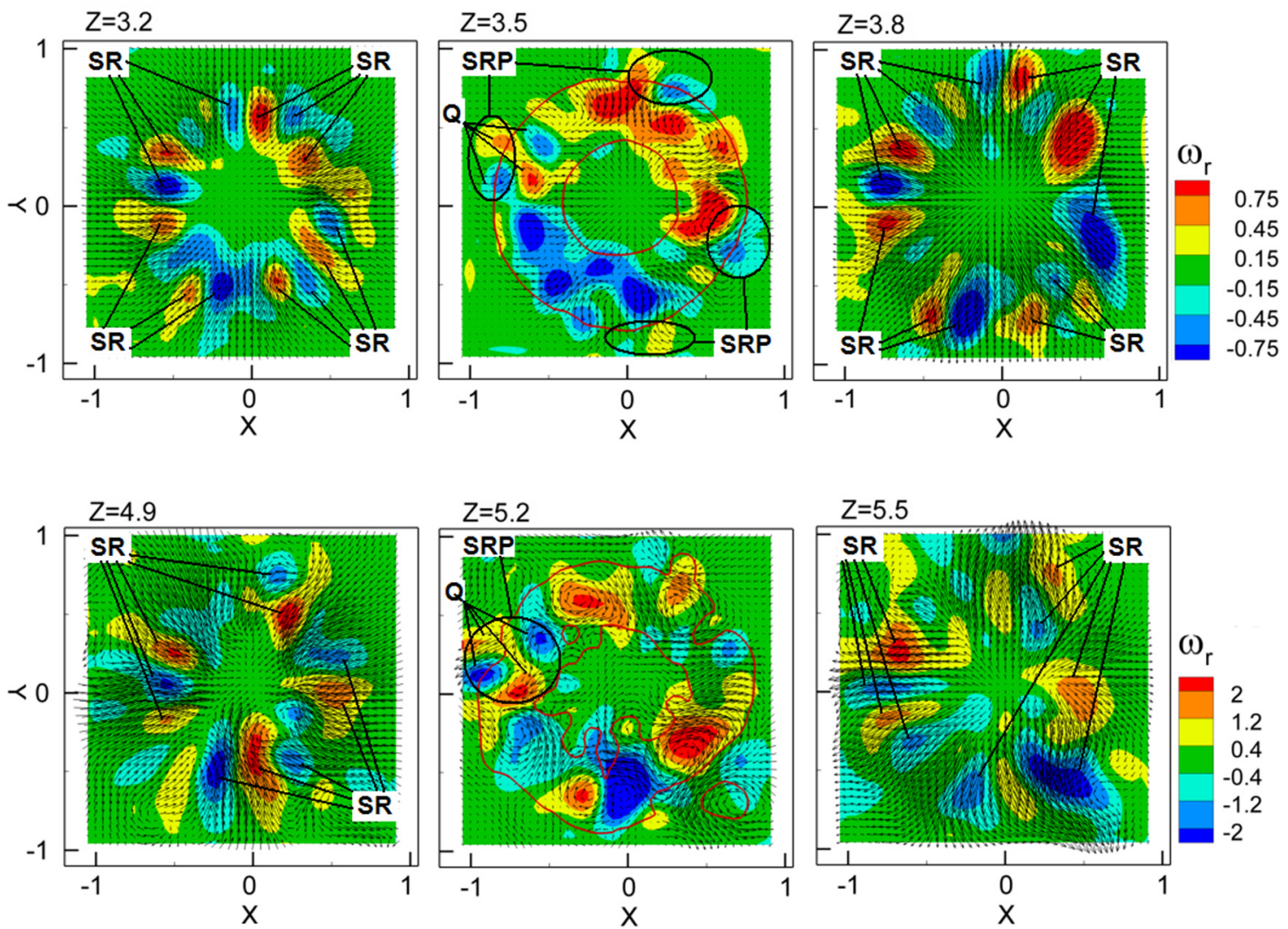

FIG. 23. (Color online) Cross-sectional iso-contours of radial vorticity $\omega_{r}$. at $t=2.44$ (top) and $t=4.92$ (bottom). $X$ - $Y$ projection of velocity vectors and with iso-line $\lambda_{2}=-0.5$. 
$t=2.44$, on the cross-section at the vortex ring trailing edge $(Z=3.2)$, a total of 13 elements with radial vorticity magnitude $0.45<\left|\omega_{r}\right|<0.75$ are located at the same radial position of "STP" vortices $(\mathrm{R}=0.6)$ and they are subjected to a rather uniform radial inward motion induced by the $\operatorname{ring}\left(V_{r}=0.3\right)$. Unlike "ST," "SR" vortices do not have a clear organization in pairs. The difference in the number of waves between azimuthal instability (4 primary waves) and the number of "SR" vortices (13) suggests that these are formed in the braid region and most likely they do not develop from the primary ring instabilities. At the vortex cross-section $(Z=3.5)$, pairs of "SR" ("SRP") are observed around the vortex ring " $3+4$." This shows regions with alternating sign of $\omega_{r}$, which may indicate that mode $\mathrm{k}=1$ is energetic. Between $Z=3.2$ and 3.5 , the outward radial motion at the ring leading edge leads to the radial displacement of "SR" vortices from $R=0.6$ to $R=0.8$. At $t=4.92$, the absolute axial vorticity of the streamwise structures increases of three times. However, the reduced azimuthal coherence of the flow results in "SR" vortices occupying a scattered position, as observed for the "ST" filaments.

The presence of 4 primary azimuthal waves and 4 streamwise vortex pairs of axial vorticity suggests a possible influence between the ring vortex and "STP" vortices. At $Z=3.5$ (Figure 21 ), the spatial modes of axial vorticity $\omega_{z}$ in the azimuthal direction are extracted for a radial position corresponding to the vortex ring $(\mathrm{R}=0.6)$ and to the streamwise structures $(\mathrm{R}=0.85)$. The corresponding modal spectra and the phase shift response are shown in Figure 24. The most energetic modes of the vortex rings are observed at low wave number $k$, such as $\mathrm{k}=2$ ("saddle" mode), 3, and 4. In the streamwise vortex, the energy is distributed over a larger range with peaks at $k=4,9,10$, and 12 . Nearly constant absolute phase shift $|\Delta \Phi|=\pi$ is found between the ring and streamwise vortices for wave numbers $\mathrm{k}=2,3,4,5,6,7,8$, and 9 . The high energy content at $k=4$ that is observed in both the spectra indicates the preferred mode of influence of streamwise pairs on vortex rings. The axial vorticity that is induced on the vortex ring is staggered in phase opposition, forming quadruplets ("Q," Figure 21). At such locations, the vortex is tilted leading to the growth of a primary hump.

The same type of analysis is conducted to investigate the influence between vortex rings and "SR" vortices. The most energetic modes of the vortex rings are found at low wave number, $k=1,2,3,4,5$, and 7. Among these, $k=1$ was already expected very energetic $(Z=3.5$, Figure 23$)$. For the "SR" structures, energy shows peaks for $k=4,6,9$, and 12. Wave numbers $\mathrm{k}=3,4$, and 7 have absolute phase shift $|\Delta \Phi|=\pi$. Although lower than the "STP" instabilities, the pronounced energy content at $k=4$ suggests that 4 streamwise pairs "SRP" have influence on vortex rings. Quadruplets of radial vorticity formed from the influence of "SRP" on ring vortices show a staggered pattern in phase opposition ("Q," Figure 23) and correspond to regions where the vortex is deformed.

\section{Chevron jet flow organization}

The early flow pattern of the chevron jet profoundly differs from the vortex ring shedding and pairing observed for the circular configuration. As shown in the temporal sequence of Figure 25, the axisymmetric ring-like coherence of the circular jet is replaced by streamwise flow structures of azimuthal ("SA"), axial ("ST"), and radial ("SR") vorticity that are formed at the nozzle exit. Vortices "ST" and "SR" are organized in counter-rotating pairs ("STP" and "SRP"), which exhibit a rather stationary behavior. Despite the absence of vortex rings, between $Z=1$ and $Z=3$, the potential core of the chevron jet still exhibits some pulsatile motion with Strouhal number $0.56<S t<0.85$, leading to instantaneous axial velocity fluctuations at jet axis of 0.12 at $Z=2$ and axial turbulence of 0.025 (Figure 13). Flow patches at axial velocity larger than $W_{\mathrm{j}}$, as shown in Figure 25 by iso-surface $W=1.05$, alternates with the ones at lower velocity in a range between 0.95 and 1.07 .

The presence of counter-rotating pairs of vortices "STP" was observed in the lobe regions between chevrons at higher Reynolds number both experimentally $3,36,37$ and computationally. ${ }^{6,7}$ Bridges and Brown ${ }^{3}$ and Opalski et al. ${ }^{36}$ showed that, when chevrons penetrate the jet core, they induce axial vorticity, resulting in evident streamwise vortices at the chevron notches. Using time-averaged stereo-PIV data at several cross-sections, Alkislar et al. ${ }^{37}$ showed the threedimensional axial development of the streamwise structures by iso-surface of axial mean vorticity. However, previous studies do not discuss the aspects related to the radial vorticity component "SR," as they were conducted by planar PIV measurements at jet axis cross-sections, which only enable the evaluation of axial vorticity. The inspection of all vorticity components was given by Xia et al. (2009), ${ }^{6}$ who conducted LES computations and showed the pattern of the vorticity magnitude. However, the discussion of the contribution from each component is lacking, in relation to the
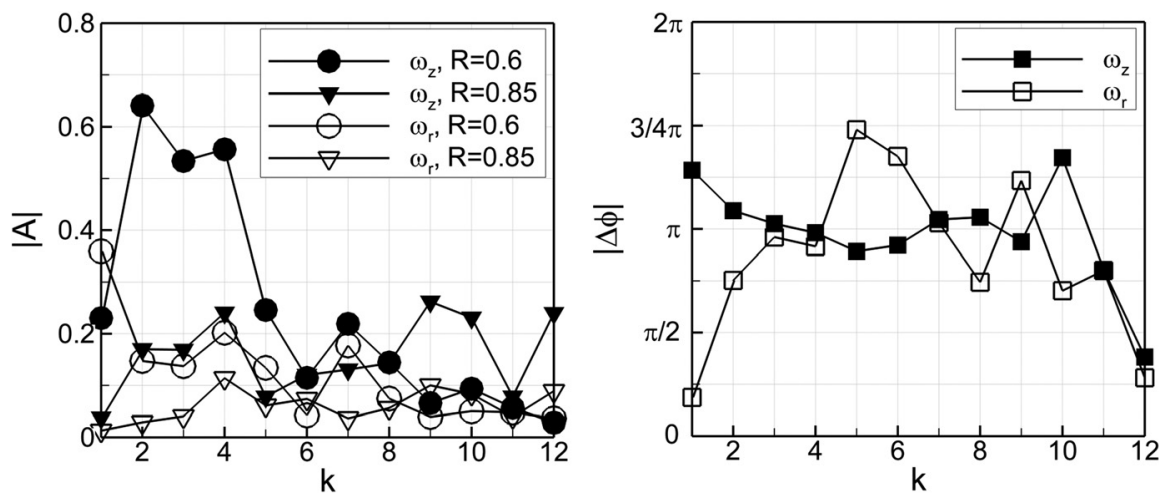

FIG. 24. Azimuthal modal spectra of $\omega_{z}$ and $\omega_{r}$ (left) and phase shift (right). 

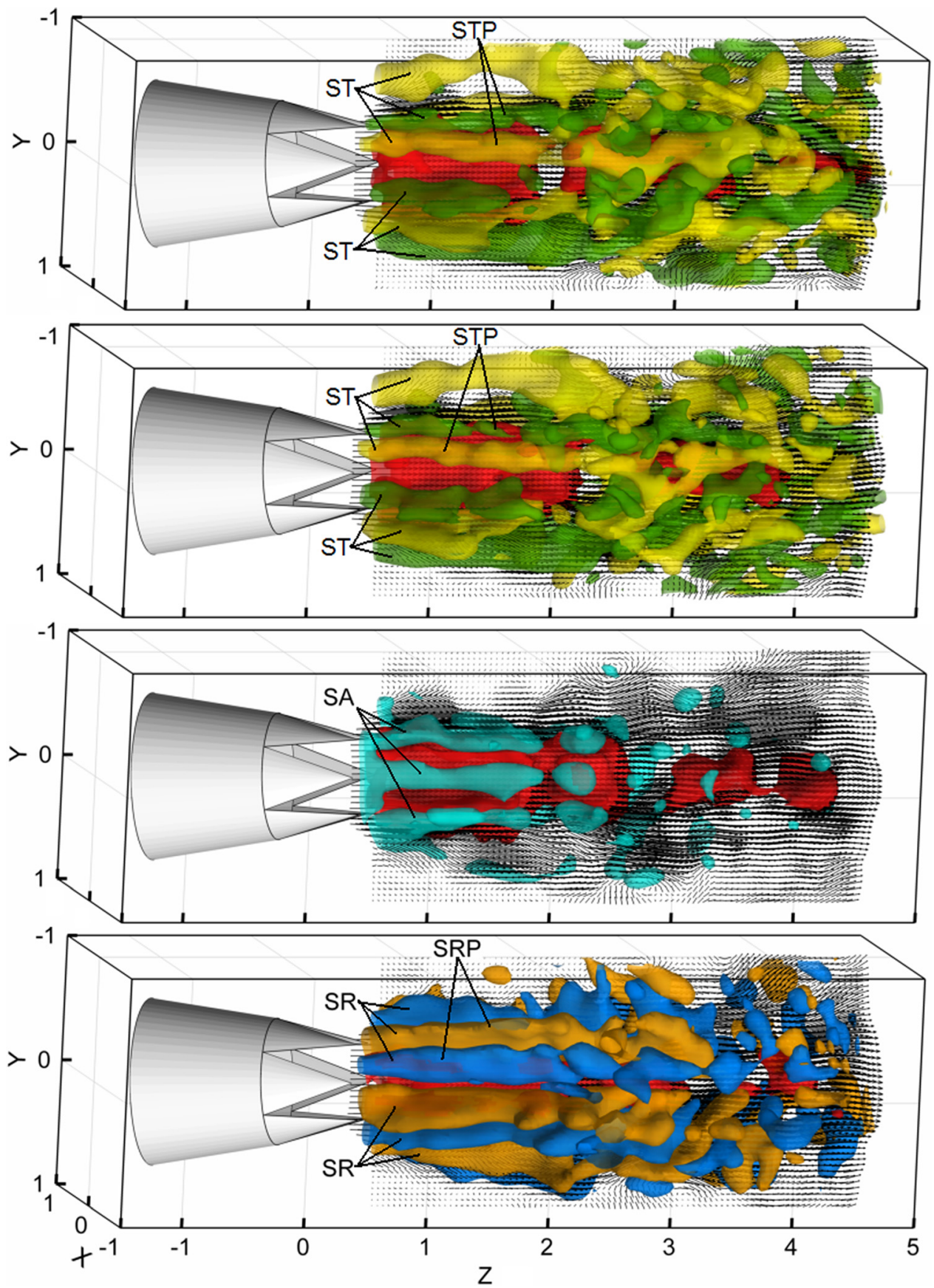

FIG. 25. (Color online) Time sequence visualization of the chevron jet. Velocity vector slice in the axial plane. Isosurfaces of axial velocity $W=1.05$ (red). In the first two snapshots, iso-surfaces $\omega_{z}=-1.2$ (green) and 1.2 (yellow); in the third snapshot iso-surfaces $\omega_{\theta}=4$; in the forth snapshot iso-surfaces $\omega_{r}=-1.2$ (blue) and 1.2 (orange). Time separation between displayed images $\Delta t=0.61$. different instability mechanisms. Also the study by Uzun and Hussaini ${ }^{7}$ focuses on the assessment of their LES simulation rather than on the flow behavior.

The cross-sectional contour plots of Figure 26 show the instantaneous velocity pattern by iso-contours of axial velocity and $X-Y$ projection of the velocity vector. At $Z=1$ and $Z=2$, circulatory patterns are observed at the chevron notch in a slightly staggered position. The coherence of these regions and the magnitude of the axial velocity in the jet core gradually decrease between $Z=2$ and $Z=4$, leading to the disappearance of the star-like pattern $(Z=4)$.

A detailed inspection of the vorticity patterns is given in Figure 27, which shows the cross-sectional contour plots of the vorticity components $\omega_{\theta}, \omega_{r}, \omega_{z}$ and vorticity magnitude $|\omega|$. At $Z=1$, a total of 6 longitudinal structures of azimuthal vorticity $\omega_{\theta}$ ("SA"), the same ones depicted in the third snapshot of Figure 25, are located in correspondence to the chevron apices and show peaks of $\omega_{\theta}=5$. Counter-rotating pairs of streamwise vortices of radial and axial vorticity, "SRP" and "STP," respectively, develop from the chevron notches, where they exhibit opposite sign and where the outward radial flow is one order of magnitude larger $\left(V_{r}=0.2\right.$ and $v_{r}{ }^{\prime}=0.03$, Figure 14) than at the chevron apex. "SRP" instabilities show peak activity of radial vorticity $\omega_{r}=4$, which is twice larger than the peaks of $\omega_{z}$ associated with "STP" structures. Compared to those of the circular jet (Figures 21 and 23), "SR" and "ST" instabilities are 50\% stronger and $40 \%$ weaker, respectively. On the other hand, absolute vorticity at the chevron apex $(|\omega|=6$, Figure 27) is comparable to that observed in the circular jet for 

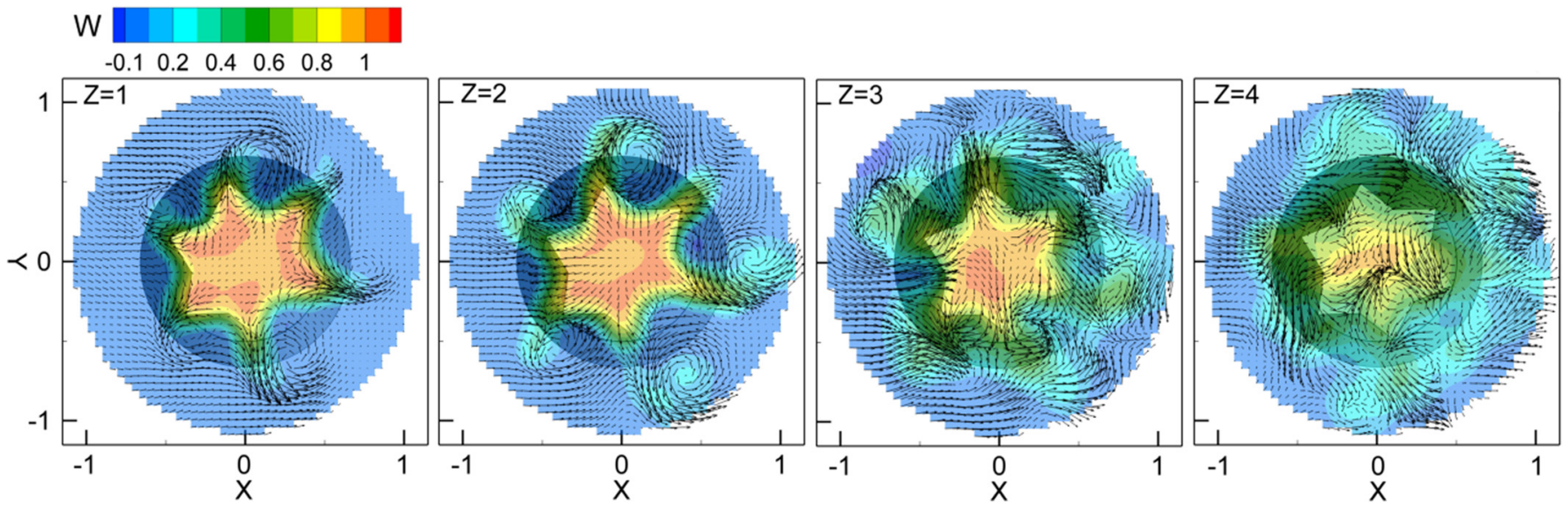

FIG. 26. (Color online) Cross-sectional iso-contours of $W ; \mathrm{X}-\mathrm{Y}$ projection of velocity vectors; $\mathrm{t}=0$.

axisymmetric vortex-rings (vortex "3," Figure 16). A decrease in $|\boldsymbol{\omega}|$ is instead observed at the chevron notches $(|\omega|<4)$.

At $Z=2.5$, branches of higher axial velocity at the notch extends to $\mathrm{R}=0.75$. Radial velocity $V_{r}$ increases to 0.25 in regions corresponding both to the notch and to the apex, where axial and radial turbulence are comparable $\left(w^{\prime}=0.1\right.$ and $v_{r}^{\prime}=0.07$, Figure 14). The increase of turbulent fluctuations amplitude is accompanied by the weakening of "SA" vortices and the movement of "ST" vortices from the notch regions, leading to the breakup of pairs. The coherence of $\omega_{r}$ instead maintains the pair organization ("SRP"). The
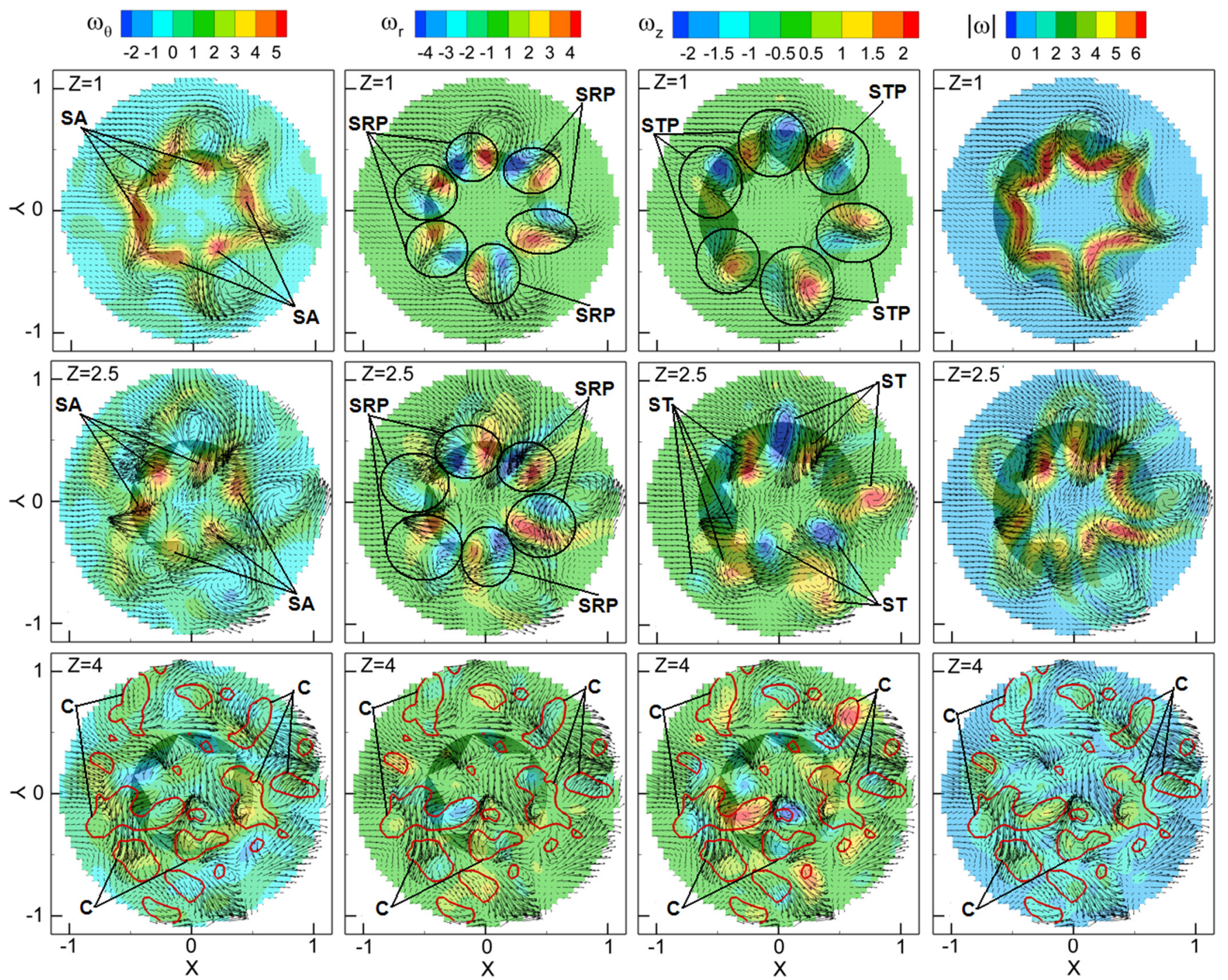

FIG. 27. (Color online) Cross-sectional iso-contour of $\omega_{\theta}, \omega_{r}, \omega_{z}$, and $|\omega|$ with iso-line $\lambda_{2}=-0.2 ; X$ - $Y$ projection of velocity vectors; $t=0$. 

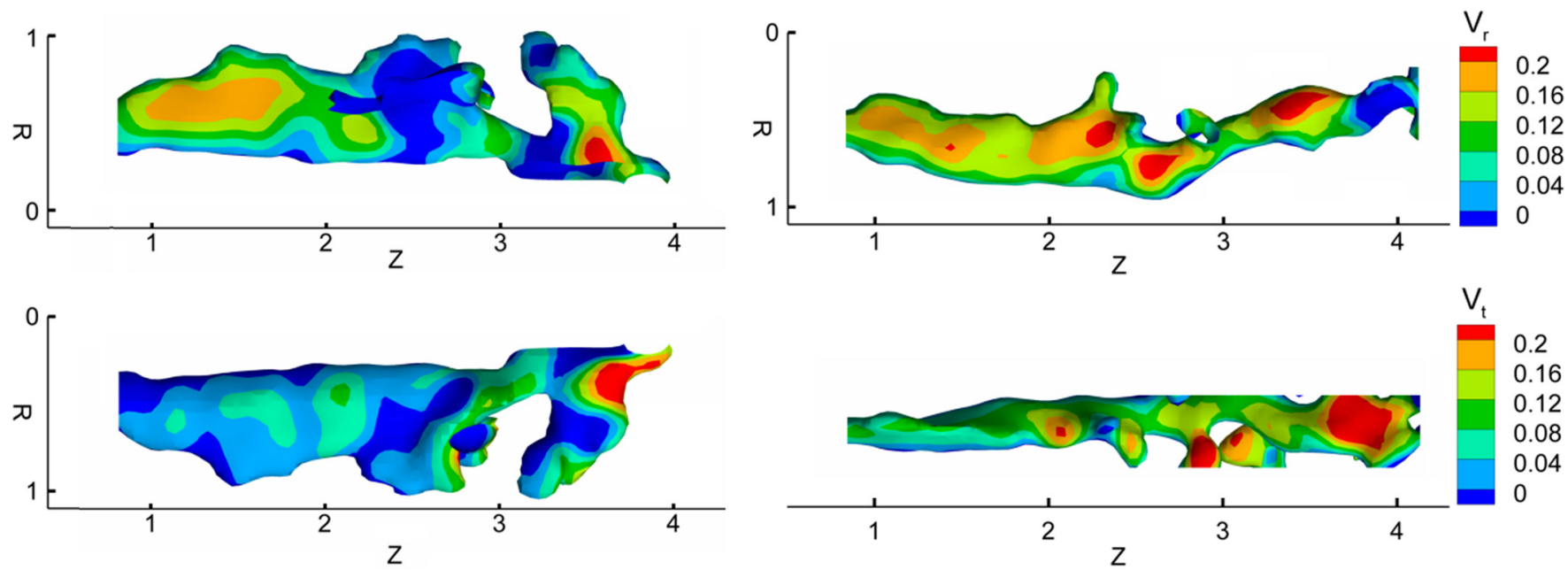

FIG. 28. (Color online) Iso-surfaces of $\omega_{r}=0.8$ (left) and $\omega_{z}=0.8$ (right) color coded with $V_{r}, V_{t} ; t=0$.

maximum absolute vorticity is concentrated at the chevron apex as seen at $Z=1$.

At $Z=4$, the contour plot of axial velocity shows that the characteristic star-like pattern has disappeared in favor of a rather axisymmetric one (Figure 26; $\mathrm{w}^{\prime}=0.07$ and $v_{r}^{\prime}=0.05$ in Figure 13) in which vortices "SA," "SRP," and "ST" no longer exist. This loss of coherence is associated with a drop of vorticity magnitude $|\boldsymbol{\omega}|$ of $30 \%$ in comparison with $Z=1$ (Figure 27). As the identification of coherent pattern at $Z=4$ becomes difficult by the vorticity vector, the $\lambda_{2}$ criterion is adopted. Iso-lines $\lambda_{2}=-0.2$ that are shown on the contour plots at $Z=4$ show that the transition region is characterized by coherent structures that will be referred as "C" vortices, discussed later in this section.

Bradshaw et $a .^{22}$ suggested that lower sound emissions in jet flows could be achieved by increasing the loss of azimuthal coherence, such as, for example, by artificial augmentation of streamwise structures with lobed nozzle. For high Reynolds number jets, the chevron configuration was shown to yield a noise reduction by experimental studies based on microphone measurements ${ }^{3,36,37}$ as well as by numerical simulations. ${ }^{6,7}$ However, the relation between the

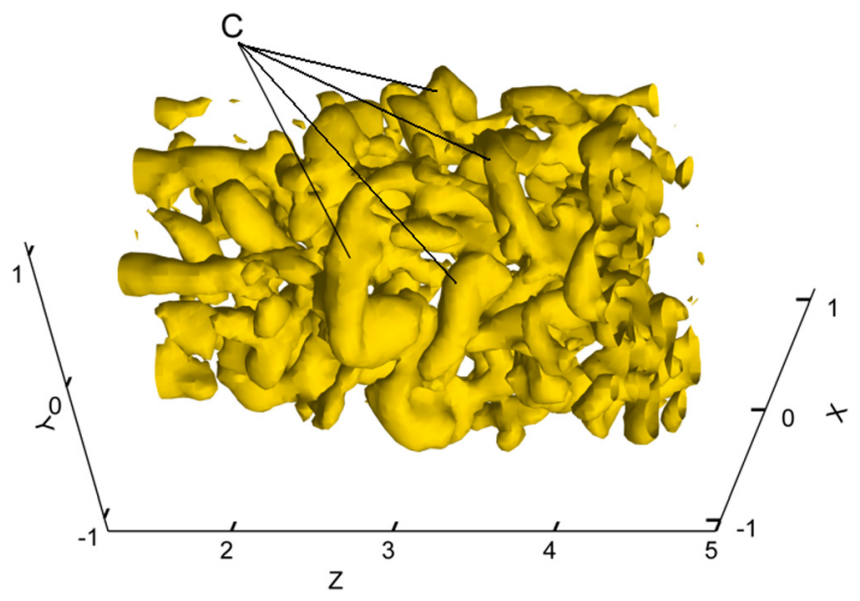

FIG. 29. (Color online) Chevron jet: "C"-like flow structures shown by isosurface of $\lambda_{2}=-0.2 ; t=0$. three-dimensional unsteady flow organization and the noise generation is still today a debated topic.

For a clearer insight in the three-dimensional development, the counter-rotating vortices "SRP" and "STP" (Figure 25) are extracted at $t=0$ and, for each of the pair, the streamwise vortex with positive sign of the related vorticity component is illustrated in Figure 28. Vortex filament "ST" extends to 4 jet diameters downstream the nozzle and, at approximately $Z=2.5$, it bends inward towards the jet core with an angle of approximately $30^{\circ}$ to the axis. The core of the streamwise vortex is stretched in the radial direction, as shown at $Z=1$ in Figure 27, with dimension of the major and minor axes of $1 / 4 D$ and $1 / 6 \mathrm{D}$ based on iso-surface $\omega_{z}=0.8$. Similar core size is observed for the "SR" structure, which develops up to $Z=2.8$ with a rather constant size of the core (Figure 27) and gradually decays between $Z=2.5$ and 4 . The velocity patterns related to the streamwise coherence are illustrated color-coding the isosurface of the streamwise vortex with radial and tangential
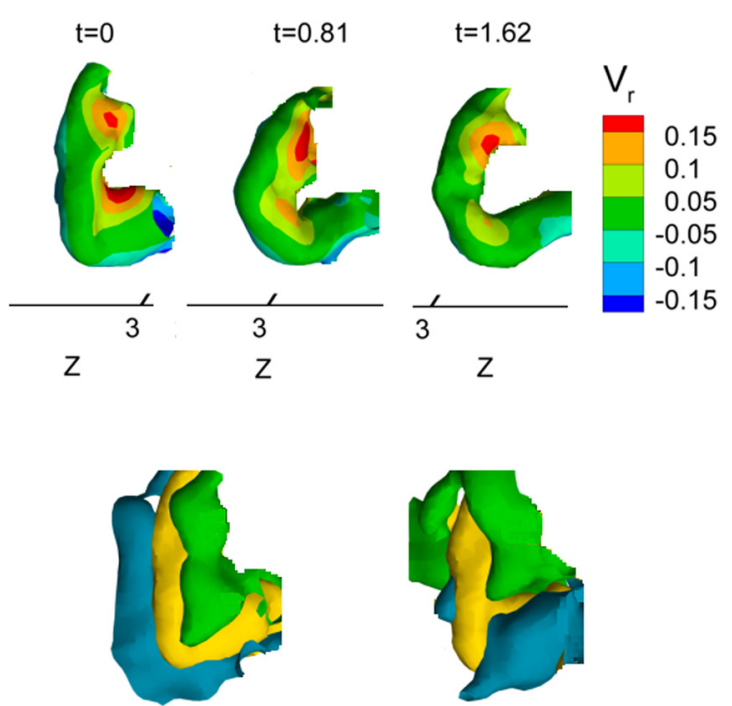

FIG. 30. (Color online) (Top) Temporal evolution of "C" structure colorcoded with $V_{r}(\Delta t=0.81)$; (bottom) iso-surface -0.05 (blue) and 0.05 (green) of $V_{r}$ (left) and $V_{t}$ (right). 


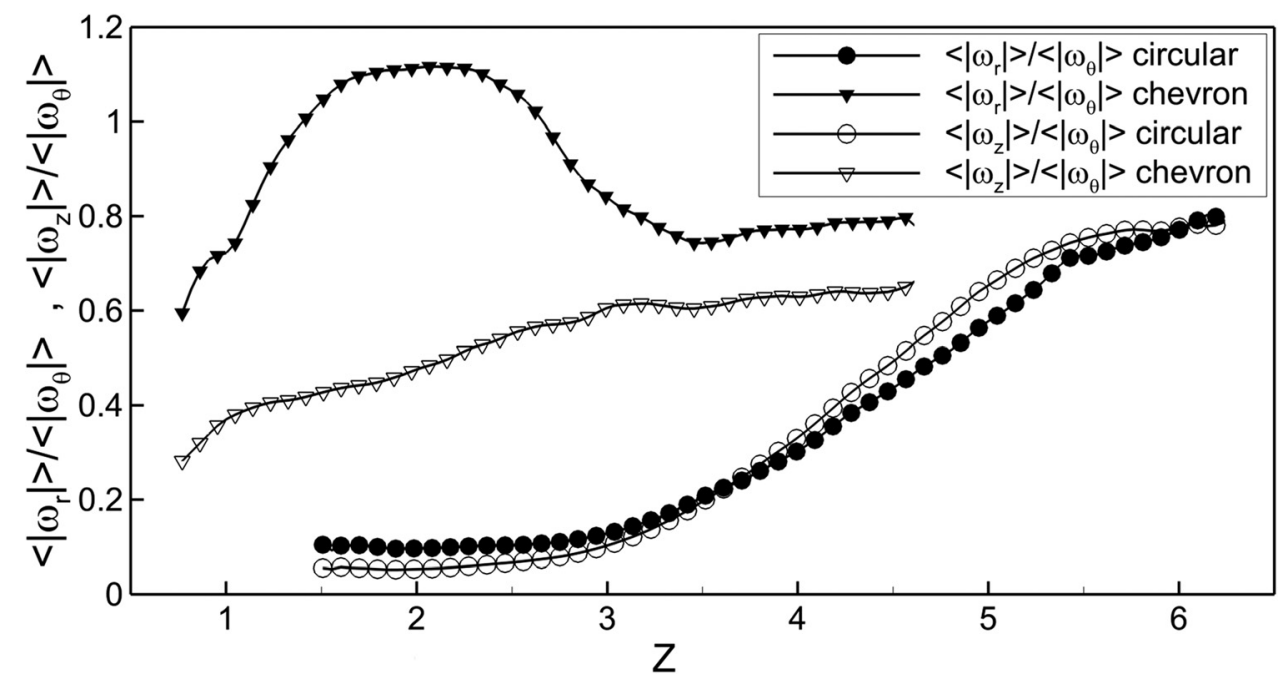

FIG. 31. Axial distribution of $\left\langle\left|\omega_{r}\right|\right\rangle \mid$ $\left\langle\left|\omega_{\theta}\right|\right\rangle$ and $\left\langle/ \omega_{z} \mid\right\rangle /\left\langle\left|\omega_{\theta}\right|\right\rangle$.

velocity components. For both "SR" and "ST," the highest levels of $V_{r}$ activity is observed on the side of the counter rotating vortex, with peaks of 0.2 , whereas the tangential velocity $V_{t}$ increases in the downstream direction reaching peaks of 0.2 at $Z>3$.

Transitional structures are characterized by a recurrent $C$ shape and are first observed where the streamwise vortices "ST" form approximately $30^{\circ}$ with the jet axis $(2.5<Z<3$, Figure 29). Here, a "C" vortex occupies a peripheral location in the shear layer $(0.5<R<1)$ that is characterized by Reynolds stress $w^{\prime} v_{r}^{\prime}=0.0025$ at both the notch and the apex of the chevron. The "legs" of the vortex are immersed in the region of higher axial velocity and, consequently, they tend to be aligned along the jet axis. This is shown in Figure 30 illustrating a temporal sequence of a " $C$ " vortex that is convected with a velocity of 0.25 . At $t=0$, the leg-to-axis angle is approximately $80^{\circ}$ and it reduces to $40^{\circ}$ at $t=1.62$. Between $Z=2.5$ and 4 , the characteristic " $C$ " shape of the vortex is conserved for a time period of 2.3, after which it breaks down. The induced flow field of a " $C$ " vortex engulfs fluid at the trailing side and ejects it at the leading side, as shown Figure 30 by the instantaneous iso-surface radial and tangential.

The normalized axial, radial, and tangential flux associated with the "C" structure can be defined as

$$
\left\{Q_{w}, Q_{r}, Q_{t}\right\}=\int_{S}\left\{W \hat{e}_{w}, V_{r} \hat{e}_{r}, V_{t} \hat{e}_{t}\right\} \cdot \overline{d S},
$$

where $S$ is the surface of the volume containing the "C" vortex, $\hat{e}_{z}, \hat{e}_{r}$, and $\hat{e}_{t}$ are the unit vector in the axial, radial, and tangential directions, $\overline{d S}$ is the vector normal to $S$. On aver-
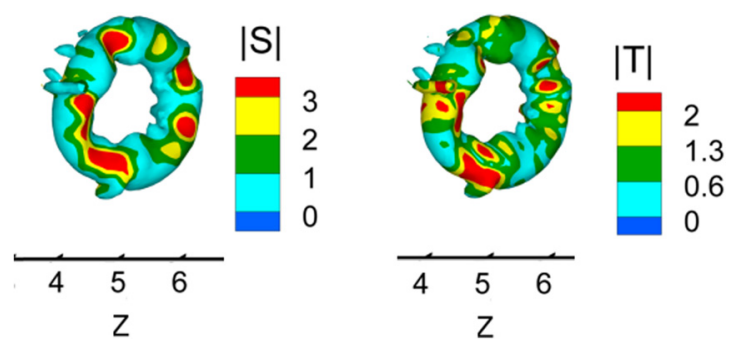

FIG. 32. (Color online) Circular jet: iso-surface $\lambda_{2}=-0.5$ of vortex " $3+4$ " color coded with $|\mathbf{S}|$ (left) and $|\mathbf{T}|$ (right) at $t=3.28$. age, it is found a net inward radial flux of 0.05 , which is comparable to the axial flux in the upward direction. In the tangential direction, instead, the flux is one order of magnitude smaller. These observations show that the "C" vortices play a role in the entrainment of the outer flow and that they may be responsible for the tilting of the streamwise structures towards the jet axis.

\section{Vorticity analysis}

The development to flow three-dimensionality in chevron and circular jets may be characterized by the ratio between the radial and the azimuthal vorticity $\left(\omega_{r} / \omega_{\theta}\right)$ and the ratio between the axial and the azimuthal components $\left(\omega_{z} / \omega_{\theta}\right)$. A statistical distribution of such ratios is shown in Figure 31, where the absolute values of vorticity components are averaged over the whole observation time and in the azimuthal direction between $R=0$ and $1\left(\left\langle\left|\omega_{r}\right|\right\rangle /\left\langle\left|\omega_{\theta}\right|\right\rangle\right.$, $\left.\left\langle\left|\omega_{z}\right|\right\rangle /\left\langle\left|\omega_{\theta}\right|\right\rangle\right)$. In the circular case, $\left\langle\left|\omega_{r}\right|\right\rangle /\left\langle\left|\omega_{\theta}\right|\right\rangle$ and $\left\langle\left|\omega_{z}\right|\right\rangle /$ $\left\langle\left|\omega_{\theta}\right|\right\rangle$ show similar trends between $\mathrm{Z}=1.5$ and 3 , region dominated by azimuthally coherent ring vortices, with the former that is twice larger than the latter (0.07) (Figure 15). They both increase in the region of vortex pairing $(2.7<Z<3.5)$, with $\left\langle\left|\omega_{z}\right|\right\rangle /\left\langle\left|\omega_{\theta}\right|\right\rangle$ that reaches $\left\langle\left|\omega_{r}\right|\right\rangle /\left\langle\left|\omega_{\theta}\right|\right\rangle$ at $\mathrm{Z}>3.6$. More downstream, $\left\langle\left|\omega_{r}\right|\right\rangle /\left\langle\left|\omega_{\theta}\right|\right\rangle$ and $\left\langle\left|\omega_{z}\right|\right\rangle /\left\langle\left|\omega_{\theta}\right|\right\rangle$ steadily increase as azimuthal instabilities and streamwise vortices "SR" and "ST" grow. At Z $=5.4$, both trends level out at 0.78 .
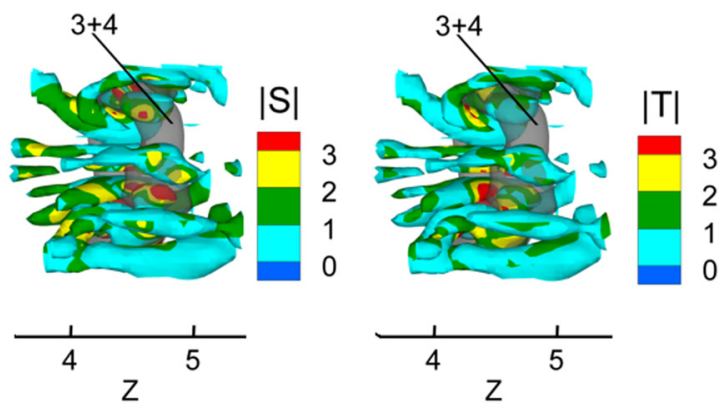

FIG. 33. (Color online) Circular jet: iso-surface $\omega_{z}=-1.2$ and 1.2 color coded with $|\mathbf{S}|$ (left) and $|\mathbf{T}|$ (right); iso-surface of vortex " $3+4 " \omega_{\theta}=4$ (grey); $t=4.1$. 


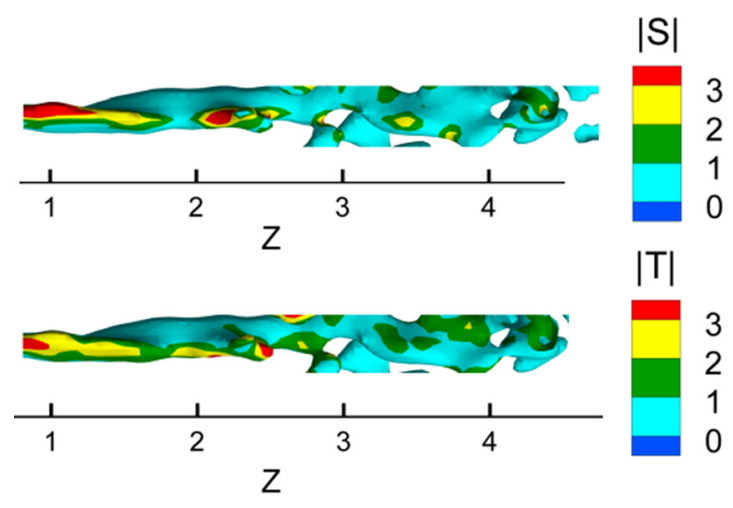

FIG. 34. (Color online) Chevron jet: iso-surfaces $\omega_{z}=0.8$ of streamwise vortex color coded with $|\mathbf{S}|$ (top) and $|\mathbf{T}|$ (bottom) at $t=0$.

In the chevron jet, $\left\langle\left|\omega_{r}\right|\right\rangle /\left\langle\left|\omega_{\theta}\right|\right\rangle$ and $\left\langle\left|\omega_{z}\right|\right\rangle /\left\langle\left|\omega_{\theta}\right|\right\rangle$ are higher than in the circular case, with the former that is always the largest at any $\mathrm{Z}$ location between 1.5 and 4.8. At $\mathrm{Z}=1$, where "SR" and "ST" structures are located at chevron notches (Figures 25 and 27) $\left\langle\left|\omega_{r}\right|\right\rangle /\left\langle\left|\omega_{\theta}\right|\right\rangle$ is 0.72, approximately twice the value shown by $\left\langle\left|\omega_{z}\right|\right\rangle /\left\langle\left|\omega_{\theta}\right|\right\rangle$. While this grows steadily, $\left\langle\left|\omega_{r}\right|\right\rangle /\left\langle\left|\omega_{\theta}\right|\right\rangle$ steeply increases to 1.1 at $\mathrm{Z}=1.6$, and then drops to 0.78 between $\mathrm{Z}=2.5$ and 3.5 , which is the region of streamwise coherence decay and " $C$ " structure formation. More downstream, $\left\langle\left|\omega_{r}\right|\right\rangle /\left\langle\left|\omega_{\theta}\right|\right\rangle$ levels out at 0.78 , whereas $\left\langle\left|\omega_{z}\right|\right\rangle /\left\langle\left|\omega_{\theta}\right|\right\rangle$ reaches a rather constant trend at 0.63 .

\section{E. Stretching and tilting of flow structures}

The normalized vortex stretching-tilting $(\omega \cdot \nabla) \mathbf{V}$ can be decoupled into a component of stretching

$$
\mathbf{S}=\left((\omega \cdot \nabla) \mathbf{V} \cdot \frac{\omega}{|\omega|}\right) \hat{\boldsymbol{\omega}}
$$

and a component of tilting

$$
\mathbf{T}=\left((\omega \cdot \nabla) \mathbf{V} \times \frac{\omega}{|\omega|}\right) \hat{\boldsymbol{\omega}},
$$

where $\hat{\omega}$ is the unit vorticity vector.

In the present approach, the vortex stretching and tilting terms are evaluated at vortex locations, which allow determin-
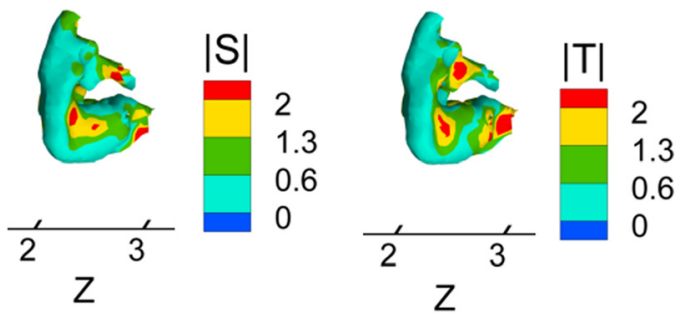

FIG. 35. (Color online) Chevron jet: iso-surfaces $\lambda_{2}=-0.2$ of "C" vortex color coded with $|\mathbf{S}|$ (left) and $|\mathbf{T}|$ (right) at $t=0$.

ing their spatial distribution within the vortex rings. In the circular jet, they increase on the vortex rings downstream of $Z=3$, especially after pairing, when azimuthal instabilities begin to grow. Figure 32 illustrates the example of vortex " $3+4$." The absolute stretching $|\mathbf{S}|$ reach peaks of 3 in correspondence to the azimuthal valley. By contrast, absolute tilting $|\mathbf{T}|$ shows peaks of 2 with pattern that are hardly associable with the vortex azimuthal deformation. Axial tilting is typically twice larger compared to the $x$ and $y$ components. On the streamwise vortices "ST," $|\mathbf{S}|$ and $|\mathbf{T}|$ are smaller than 1 on the leading side of the vortex ring, whereas they are maximum (3.5, Figure 33) on the longitudinal structures located within the vortex ring. On the trailing side of the vortex ring, instead, $|\mathbf{S}|$ is 2.5 and $|\mathbf{T}|$ is 1.5. Comparable peak values are observed for "SR" vortices, which are not illustrated.

In the chevron jet, the streamwise structure "ST" are highly stretched and tilted on the side of the nozzle between $Z=0.8$ and $Z=2$, as depicted in Figure 34. $|\mathbf{S}|$ and $|\mathbf{T}|$ reach peak values of 3 and they substantially decrease in the downstream direction, with a drop of $30 \%$ in the regions corresponding to the "C" vortices. The upstream and downstream sides are characterized by $|\mathbf{S}|=2.2$ and $|\mathbf{T}|=2.2$ and a decrease to values smaller than 0.5 on the lateral side which is embedded in the region of lower axial velocity (Figure 35). Similar patterns are observed for the streamwise structures "SR," which are not illustrated.

Absolute stretching $|\mathbf{S}|$ and absolute tilting $|\mathbf{T}|$ are averaged over the whole observation time, in the azimuthal direction and between $\mathrm{R}=0$ and 1 to statistically compare the axial activity of the chevron jet with that of the circular configuration. The resulting statistical trends $\langle|\mathbf{S}|\rangle$ and $\langle|\mathbf{T}|\rangle$ are illustrated in Figure 36. In the chevron jet, between the nozzle exit

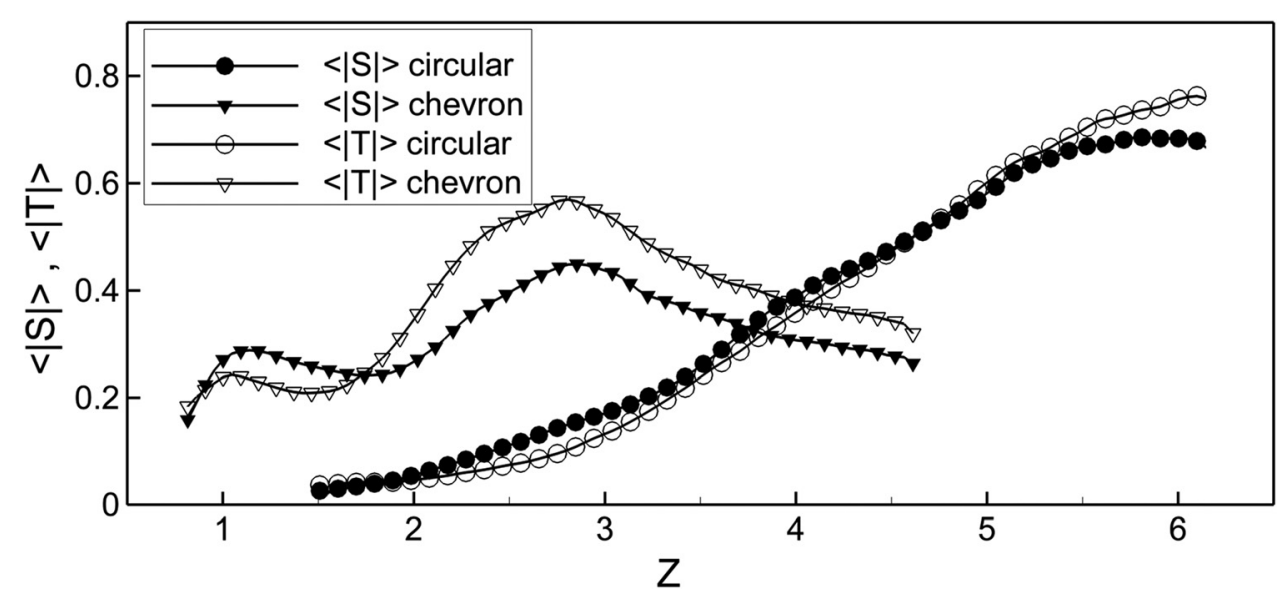

FIG. 36. Space-time average of $|\mathbf{S}|$ and $|\mathbf{T}|$. 

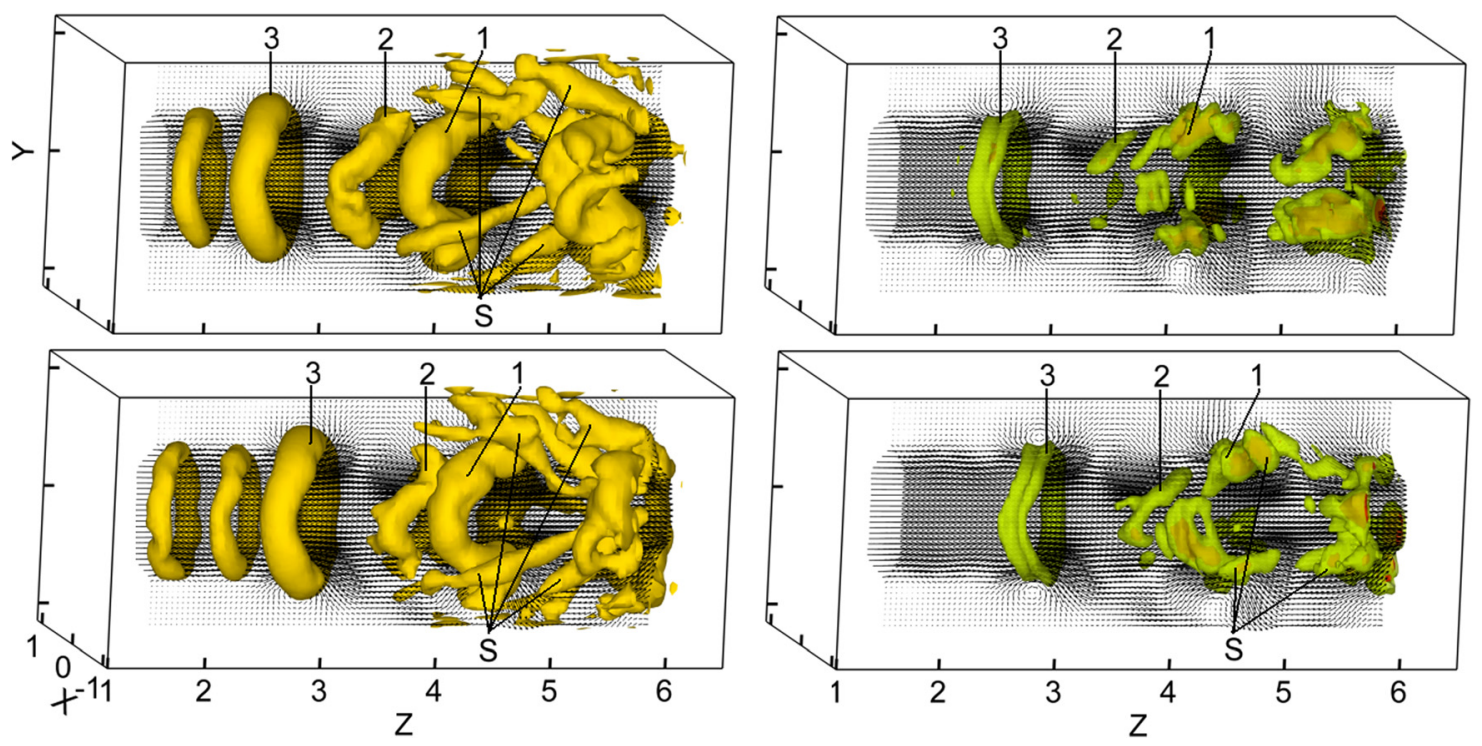

FIG. 37. (Color online) Circular jet: (left) subsequent snapshots of iso-surfaces of $\lambda_{2}=-0.5$ and (right) corresponding iso-surfaces of $\mathrm{L}=30$ (green) and 50 (red).

and $Z=2,\langle|\mathbf{S}|\rangle$ and $\langle|\mathbf{T}|\rangle$ reach peaks of 0.28 and 0.24 , respectively, which are approximately 5 times larger what observed for the circular configuration. In the chevron jet, peak activity of stretching and tilting $(\langle|\mathbf{S}|\rangle=0.45$ and $\langle|\mathbf{T}|\rangle=0.57)$ is found at $Z=2.8$, where the streamwise vortices "ST" and "SR" begin to decay and " $C$ " structures are formed. Further downstream, instead they decrease of approximately $30 \%$ at $Z=4$.

In the circular case, $\langle|\mathbf{S}|\rangle$ and $\langle|\mathbf{T}|\rangle$ show a steep increase for $Z>3$, where the flow is characterized by vortex pairing and by fast growth of azimuthal instabilities and streamwise filaments. Stretching and tilting are larger than in the chevron for $Z>4.5$ and $Z>5$, respectively, where they increase to $\langle|\mathbf{S}|\rangle=0.68$ and $\langle|\mathbf{T}|\rangle=0.74$.

\section{F. Relation to sound production}

The phenomenological aspects of the large-scale flow fluctuations have been so far discussed in relation to the unsteady and three-dimensional behavior at transition. Early experimental investigations on circular-jet noise showed that the production of acoustic waves is mainly related to largescale structures, e.g., vortex rings, ${ }^{9,22,33}$ indicating the most radiating events in the Strouhal number range between 0.2 and $1 .{ }^{32}$ Bradshaw et al. ${ }^{22}$ suggested that the loss of azimuthal coherence by artificial augmentation of streamwise structures, such as by lobed nozzles, could lead to lower sound emissions, which was reported in later studies for chevron jets at high Reynolds number. ${ }^{3,6,7,36,37}$ For hot jets, Dahan et al. ${ }^{69}$ experimentally showed that $50 \%$ of the farfield acoustic energy could be addressed to the dynamics of the coherent structures. Jordan et al. ${ }^{70}$ used POD on LES simulation of a jet at $\mathrm{M}=0.9$ and $\mathrm{Re}=3600$ and showed that $12 \%$ of the far-field sound is related to convected axisymmetric vortex-rings, whereas over $48 \%$ is due to the process of disruption of vortex ring involving vortex-ring azimuthal instabilities and streamwise vortices.

In some recent studies, PIV measurements have been used in combination with aeroacoustic analogies to characterize noise sources. A statistical approach was used, for instance, by Seiner et $a l .{ }^{41}$ and Fleury et $a l .{ }^{43}$ who evaluated Lighthill stress tensor by means of correlation functions. In the investigation of vortex-pairing sound, Schram et al. ${ }^{42}$ applied phase-locked planar PIV to a conservative formulation of vortex sound theory for axisymmetric flow, observing good agreement between the acoustic prediction and microphone measurements. The potential and relevance of a method that combines PIV with acoustic analogies have found recent acknowledgements of recent review articles, ${ }^{35,47}$ motivating its application in this work.

The role of coherent vortices in the local activity of acoustic sources is hereby explored recalling Powell's aeroacoustic analogy. ${ }^{56}$ Although the current experiment does not
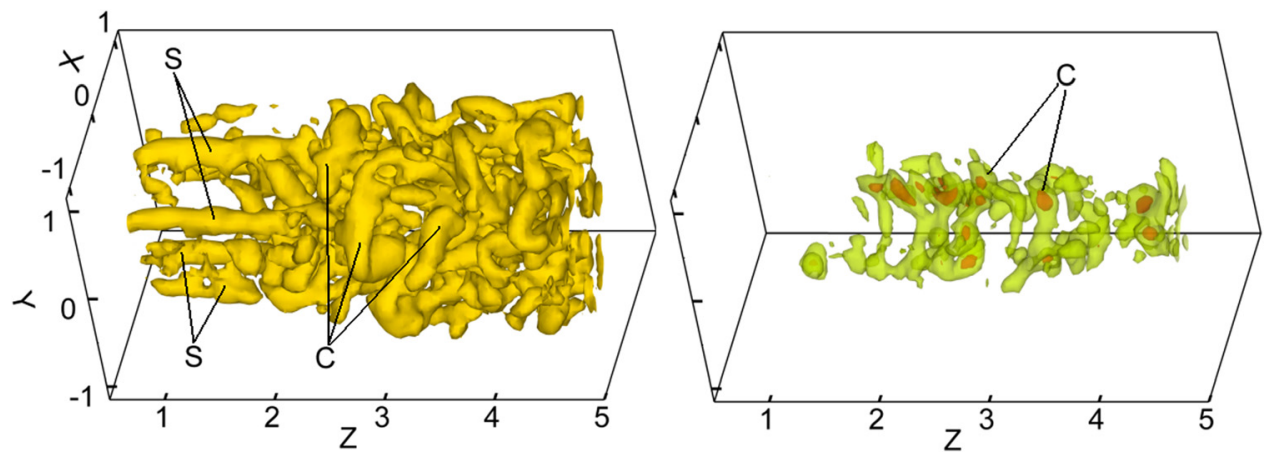

FIG. 38. (Color online) Chevron jet: (left) snapshot of iso-surfaces of $\lambda_{2}=-0.2$ and (right) corresponding isosurfaces of $\mathrm{L}=30$ (green) and 50 (red). 


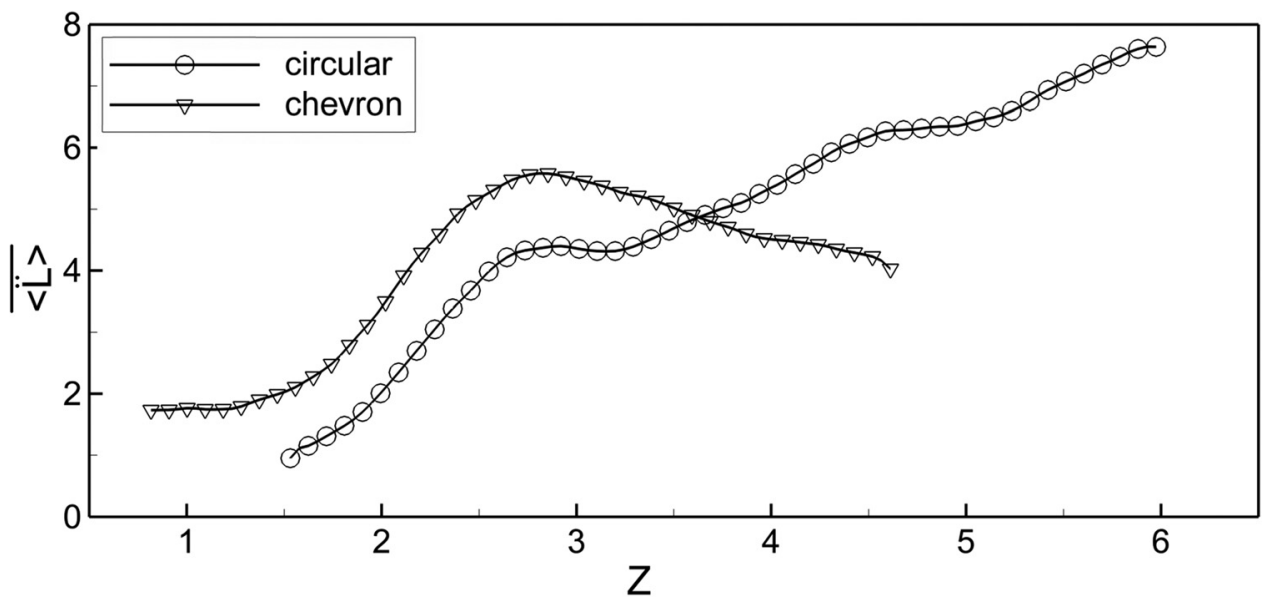

FIG. 39. Space-time average of L.

enable to cover the entire region of acoustic production, neither to resolve all the spatial scales at which acoustic fluctuations are produced, the contributions due to vortex rings, streamwise vortices, and "C" structures are explored.

In Powell's analogy, the Lamb vector, which is defined as $\mathbf{L}^{*}=\boldsymbol{\omega}^{*} \times \mathbf{v}^{*}$, is part of the integrand term and accounts for the flow state. For the linear property of the integral function, the integrand can be rewritten as the integral of the second time derivative of the normalized Lamb vector $\left(\mathbf{L}=\left(d^{2} \mathbf{L}^{*} / d t^{* 2}\right) D^{3} / W_{j}^{4}\right)$. This property clearly requires three-dimensional and time-resolved information, which, except for the current experiment, may only be considered available from numerical simulations (DNS or LES). A survey on the spatio-temporal pattern of

$$
\mathbf{L}=\left|\frac{\partial^{2}}{\partial t^{2}} \mathbf{L}\right|=\left[\left(\frac{\partial^{2}}{\partial t^{2}} L_{X}\right)^{2}+\left(\frac{\partial^{2}}{\partial t^{2}} L_{Y}\right)^{2}+\left(\frac{\partial^{2}}{\partial t^{2}} L_{Z}\right)^{2}\right]^{1 / 2}
$$

not yet available in literature, is discussed in relation largescale structures that are identified by the $\lambda_{2}$-criterion. As the radial and axial vorticity components cannot be distinguished ("ST" and "SR") by such criterion, streamwise structures will be more generically referred to as " $\mathrm{S}$ " vortices.

In the circular jet, as shown in Figure 37, illustrating two subsequent snapshots of the pairing of vortex " 1 " and " 2 ," the production of $\mathrm{L}$ is observed downstream of $Z=3$, where vortex rings are characterized by $\mathrm{L}=30$ (" 3 "). Peak activity of 50 is observed corresponding to the rise of azimuthal instabilities ("1" and "2") and streamwise structures ("S").

In the chevron jet (Figure 38), streamwise vortex pairs are not characterized by major production of L. This, instead, increases to 30 between $R=0$ and $R=0.7$, at approximately $Z=2.5$, where the streamwise structures begin to decay and "C" vortices are formed and it also shows peaks of 50 .

The average of $\mathrm{L}$ over the entire observation time and along radial $(0<\mathrm{R}<1)$ and azimuthal directions $\overline{(\langle\ddot{\mathrm{L}}\rangle})$ is used as a criterion to localize the most active regions where the fluctuations of pressure will result into noise (Figure 39). In both configurations, $\overline{\langle\ddot{\mathrm{L}}\rangle}$ increases considerably starting from $Z=1.5$. In the chevron jet, $\overline{\langle\ddot{\mathrm{L}}\rangle}$ shows a pronounced activity of 5.5 at $Z=2.8$, where "C" structure are formed and streamwise-vortex pairs ("S") lose coherence. Compared to the value found at $Z=0.8(1.8)$, the peak activity is 3 times larger. In the circular case, instead, $\langle\ddot{\mathrm{L}}\rangle$ grows up to 4.3 in the region of pairing $(Z=3)$, where the activity is 4 times larger then at $Z=1.5$. Similarly to what seen for the stretching and tilting, in the chevron configuration, $\overline{\langle\ddot{\mathrm{L}}\rangle}$ decreases for $Z>2.8$ with a $30 \%$ drop at $Z=4.7$. In the circular configuration, $\langle\ddot{\mathrm{L}}\rangle$ gradually increases of $40 \%$ between $Z=3.5$ and $Z=6$, where azimuthal instabilities and streamwise filament are observed.

\section{CONCLUSIONS}

The three-dimensional behavior of flow transition in circular and 6-chevron jets at $R e=5000$ has been studied by experiments conducted in a tailored-water jet facility with time-resolved tomographic PIV. The vortex topological analysis using vorticity and $\lambda_{2}$-criterion shows differences in the large-scale flow organization between the circular and the chevron configuration.

In the circular case, the flow is characterized by a pulsatile motion leading to the shedding of vortex rings with a Strouhal number of 0.72 . Between 2.75 and 3.5 jet diameters, vortex rings pair with a characteristic frequency of $S t=0.36$. This process is characterized by peaks of axial and radial turbulence in the shear layer $\left(w^{\prime}=0.2\right.$ and $\left.v_{r}^{\prime}=0.21\right)$. The flow axial strain leads to the formation of three-dimensional patterns such as vortex-azimuthal instabilities and counterrotating streamwise vortices of axial and radial vorticity ("ST" and "SR," respectively). On average of 4 primary azimuthal waves grow on the vortex ring, leading to in-plane and out-of-plane deformation. Streamwise vortices "ST" and "SR" are distributed around the vortex rings with an angle of $30^{\circ}-40^{\circ}$ to the jet axis and they move with a characteristic inward-outward radial motion that is induced by the passage of the vortex rings. The axial vorticity peaks of "ST" $\left(\omega_{z}=3.5\right)$ are typically $50 \%$ larger than the radial ones of "SR." At $Z=3.2$, a total of 11 streamwise vortex "ST" develop from the braid region and typically form 4 main streamwise pairs that interact with the vortex ring. The modal decomposition of axial vorticity shows that, in the azimuthal direction, the vortex rings and the streamwise 
vortices are characterized by energy peak at wave number $k=4$ with a phase shift of $\pi$. The axial vorticity induced by streamwise pairs on the vortex ring is staggered in phase opposition at the regions of interaction, where primary instabilities grow.

In the chevron jet, the column is characterized by a pulsitile motion $(0.56<S t<0.85)$ and the axisymmetric ringlike coherence observed for the circular jet is replaced by streamwise flow structures of azimuthal ("SA"), axial ("ST"), and radial ("SR") vorticity that are formed at the nozzle exit. "SA" filaments develop from the nozzle apexes and show peaks of $\omega_{\theta}=5$. By contrast, 6 counter-rotating pairs of streamwise vortices "STP" and "SRP" are formed at the nozzle exit in a position corresponding to the chevron notch, where the radial velocity is maximum $\left(v^{\prime}{ }_{r}=0.25\right.$ at $Z=1$ ), and they extend downstream up to $Z=4$. The maximum of radial vorticity associated with "SRP" structures $\left(\omega_{r}=4\right)$ is twice larger than that observed for the "STP" ones. However, "SRP" and "STP" are, respectively, 50\% stronger and $40 \%$ weaker than the corresponding structures of the circular jet. Between $Z=2.5$ and $Z=3$, the shear layer $(0.5<R<1)$ is organized in " $C$ " shaped vortices which, inducing inward radial flow, may be responsible for the tilting of the streamwise structures towards to the jet axis.

The development to flow three-dimensionality in the jetaxis direction is statistically characterized by space-time average of the ratio between axial and azimuthal vorticity and the ratio between radial and azimuthal vorticity. In the circular jet, these ratios steadily increase as vortices pair and azimuthal instabilities and streamwise vortices grow. Instead, in the chevron configuration, where streamwise structures develop from the nozzle exit, vorticity ratios reach values more than 5 times larger than in the circular jet and they level out with the formation of "C" structures.

The stretching and tilting fields are analyzed in relation to the coherent structures. In the circular case, instantaneous peak activity is observed with the rise of three-dimensional patterns and is associated with azimuthal instabilities and on streamwise vortices. Comparable peak activity $(|\mathbf{S}|=3$, $|\mathbf{T}|=3$ ) is observed on the streamwise filaments and "C" vortices for the chevron jet. A statistical characterization based on a space-time average shows that, in the chevron jet, the maximum of stretching and tilting corresponds to the region, where " $C$ " structures are formed and interact with streamwise filaments $(2.5<Z<3)$. In the circular case, instead, stretching and tilting fields gradually increase where the flow has three-dimensional patterns $(Z>3)$, reaching values larger than in the chevron jet at $Z=6$.

The relation between coherent flow structures and the instantaneous acoustic production is investigated recalling Powell's analogy. Noise sources are characterized by the second time derivative of the Lamb vector. Its spatiotemporal evolution is visually compared to that of the vortices, which are detected by $\lambda_{2}$-criterion, to identify largescale patterns involved in the acoustic generation. In both the jets, major source activity is observed downstream of 2 jet diameters. In the circular jet, peaks are associated with the vortex pairing, azimuthal instabilities, and streamwise filaments. In the chevron jet, peak activity is observed during the process of streamwise vortex decay and $\mathrm{C}$ structure formation. A statistical characterization of Lamb vector second time derivative shows a peak at $\mathrm{Z}=2.8$ for the chevron and for the circular configuration a gradual increase in the axial direction.

\section{ACKNOWLEDGMENTS}

This work was conducted as part of the FLOVIST project (Flow Visualization Inspired Aeroacoustics with Time Resolved Tomographic Particle Image Velocimetry) funded by the European Research Council (ERC), Grant No. 202887. The authors kindly acknowledge the contribution of Kristof Bryon in the design of the water jet facility and realization of the tomographic experiments.

${ }^{1}$ C. M. Ho and E. Gutmark, "Vortex induction and mass entrainment in a small aspect-ratio elliptic jet," J. Fluid Mech. 179, 383 (1987).

${ }^{2}$ M. El Hassan and A. Meslem, "Time-resolved stereoscopic particle image velocimetry investigation of the entrainment in the near field of circular and daisy shaped orifice jets," Phys. Fluids 22, 035107 (2010).

${ }^{3}$ J. Bridges and C. A. Brown, "Parametric testing of chevrons on single flow hot jets," Technical Report No. 213107, NASA, 2004.

${ }^{4} \mathrm{~J}$. B. Freund, "Noise sources in a low-Reynolds-number turbulent jet at Mach 0.9," J. Fluid Mech. 438, 277 (2001).

${ }^{5}$ B. J. Boersma, "Large eddy simulation of the sound field of a round turbulent jet," Theor. Comput. Fluid Dyn. 19, 161 (2005).

${ }^{6}$ H. Xia, P. G. Tucker, and S. Eastwood, "Large-eddy simulations of chevron jet flows with noise predictions," Int. J. Heat Fluid Flow 30, 1067 (2009).

${ }^{7}$ A. Uzun and M. Y. Hussaini, "Simulation of noise generation in nearnozzle region of a chevron nozzle jet," AIAA J. 47, 1793 (2009).

${ }^{8}$ H. A. Becker and T. A. Massaro, "Vortex evolution in a round jet," J. Fluid Mech. 31, 435 (1968).

${ }^{9}$ S. C. Crow and F. H. Champagne, "Orderly structure in jet turbulence," J. Fluid Mech. 48, 547 (1971).

${ }^{10}$ P. E. Dimotakis, R. C. Miake-Lye, and D. A. Papantoiou, "Structure dynamics of round turbulent jets," Phys. Fluids 26, 3185 (1983).

${ }^{11}$ C. O. Paschereit, D. Oster, T. A. Long, H. E. Fiedler, and I. Wygnaski, "Flow visualization of interactions among large coherent structures in an axisymmetric jet," Exp. Fluids 12, 189 (1992).

${ }^{12}$ D. Liepmann and M. Gharib, "The role of streamwise vorticity in the near field entrainment of round jets," J. Fluid Mech. 245, 643 (1992).

${ }^{13}$ B. Ganapathisubramani, K. E. Longmire, and I. Marusic, "Investigation of three dimensionality in the near field of a round jet using stereo PIV," J. Turbul. 3, 1 (2002).

${ }^{14}$ T. Matsuda and J. Sakakibara, "On the vortical structure in a round jet," Phys. Fluids 17, 025106 (2005).

${ }^{15}$ K. P. Lynch and B. S. Thurow, "POD analysis of 3D-flow visualization images of a circular jet with Reynolds number 9500," in 39th Fluid Dynamics Conference, San Antonio, TX, USA, 2009.

${ }^{16}$ M. P. Wernet, "Temporally resolved PIV for space-time correlations in both cold and hot jet flows," Meas. Sci. Technol. 18, 1387 (2007).

${ }^{17}$ T. Hori and J. Sakakibara, "High-speed scanning stereoscopic PIV for 3D vorticity measurement in liquids," Meas. Sci. Technol. 15, 1067 (2004).

${ }^{18} \mathrm{C}$. Bruecker and W. Althaus, "Study of vortex breakdown by particle tracking velocimetry (PTV). Part 3: time-dependent structure and development of breakdown-modes," Exp. Fluids 18-3, 174 (1995).

${ }^{19}$ G. E. Elsinga, F. Scarano, B. Wieneke, and B. W. van Oudheusden, "Tomographic particle image velocimetry," Exp. Fluids 41, 933 (2006).

${ }^{20}$ M. Khashehchi, G. E. Elsinga, A. Ooi, J. Soria, and I. Marusic, "Studying invariants of the velocity gradient tensor of a round turbulent jet across the turbulent/nonturbulent interface using TOMO PIV," in 15th Int. Symp. on Applications of Laser Techniques to Fluid Mechanics, Lisbon, Portugal, 2010.

${ }^{21}$ K. Staack, R. Geisler, A. Schröder, and D. Michaelis, "3D3C coherent structure measurement in a free turbulent jet," in 15th Int. Symp. on Applications of Laser Techniques to Fluid Mechanics, Lisbon, Portugal, 2010.

${ }^{22}$ P. Bradshaw, D. H. Ferriss, and R. F. Johnson, "Turbulence in the noise producing region of a circular jet,” J. Fluid Mech. 19, 591 (1964). 
${ }^{23}$ C. D. Winant and F. K. Browand, "Vortex pairing: The mechanism of turbulent mixing layer growth at moderate Reynolds number," J. Fluid Mech. 63, 237 (1974).

${ }^{24}$ K. B. M. Q. Zaman and A. K. M. F. Hussain, "Vortex pairing in a circular jet under controlled excitation. Part 1: General jet response," J. Fluid Mech. 101, 449 (1980).

${ }^{25} \mathrm{C}$. Schram, "Aeroacoustic of subsonic jets: Prediction of the sound produced by vortex pairing based on particle image velocimetry," Ph.D. dissertation (Eindhoven University, the Netherlands, 2003), http:// web.phys.tue.nl/nl/de_faculteit/capaciteitsgroepen/transportfysica/fluid_dynamics_lab/publications/gdy/.

${ }^{26}$ S. E. Widnall and J. P. Sullivan, "On the stability of vortex rings," Proc. R. Soc. London 332, 335 (1973).

${ }^{27}$ A. J. Yule, "Large structure in the mixing layer of a round jet," J. Fluid Mech. 89, 413 (1978).

${ }^{28}$ R. W. Paterson, "Turbofan mixer nozzle flow field: A benchmark experimental study," ASME J. Eng. Gas Turbines Power 106, 692 (1984).

${ }^{29}$ K. B. M. Q. Zaman and G. Raman, "Reversal in spreading of a tabbed circular jet under controlled excitation," Phys. Fluids 9, 3733 (1997).

${ }^{30}$ M. B. Alkislar, A. Krothapalli, and L. M. Lourenço, "Structure of a screeching rectangular jet: A stereoscopic particle image velocimetry study," J. Fluid Mech. 489, 121 (2003).

${ }^{31}$ M. El Hassan, A. Meslem, and K. Abed-Meraim, "Experimental investigation of the flow in the near-field of a cross-shaped orifice jet," Phys. Fluids 23, 045101 (2011).

${ }^{32} \mathrm{~A}$. Michalke and H. V. Fuchs, "On turbulence and noise of an axisymmetric shear flow," J. Fluid Mech. 70, 179 (1975).

${ }^{33} \mathrm{C}$. J. Moore, "The role of shear layer instability waves in jet exhaust noise," J. Fluid Mech. 80, 321 (1977).

${ }^{34}$ J. I. Hileman, B. S. Thurow, E. J. Caraballo, and M. Samimy, "Large-scale structure evolution and sound emission in high-speed jets: Real-time visualization with simultaneous acoustic measurements," J. Fluid Mech. 544, 277 (2005).

${ }^{35}$ P. Jordan and Y. Gervais, "Subsonic jet aeroacoustics: Associating experiment, modeling and simulation," Exp. Fluids 44, 1 (2008).

${ }^{36}$ A. B. Opalski, M. P. Wernet, and J. E. Bridges, "Chevron nozzle performance characterization using stereoscopic DPIV," AIAA Paper No. 444 (2005).

${ }^{37}$ M. B. Alkislar, A. Krothapalli, and G. W. Butler, "The effect of streamwise vortices on the aeroacoustics of a Mach 0.9," J. Fluid Mech. 578, 139 (2007).

${ }^{38}$ R. Thomas, K. Kinzie, and S. Pao, "Computational analysis of a pylonchevron core nozzle interaction," in 7th AIAA/CEAS Aeroacoustics Conference, Maastricht, the Netherlands, 2001.

${ }^{39}$ S. F. Birch, D. A. Lyubimov, V. P. Maslov, and A. N. Secundov, "Noise prediction for chevron nozzle flows," in 12th AIAA/CEAS Aeroacoustics Conference, 27th AIAA Aeroacoustics Conference, Cambridge, MA, USA, 2006.

${ }^{40}$ F. Coiffet, P. Jordan, J. Delville, Y. Gervais, and F. Ricaud, "Coherent structures in subsonic jets: A quasi irrotational source mechanism?" Int. J. Aeroacoust. 5, 67 (2006).

${ }^{41}$ J. M. Seiner, L. Ukeiley, and M. K. Poton, "Jet noise source measurements using PIV," in 5th AIAA/CEAS Aeroacoustic conference, Bellevue, Great Seattle, USA, 1999.

${ }^{42}$ C. Schram, S. Taubitz, J. Anthoine, and A. Hirschberg, "Theoretical/empirical prediction and measurement of the sound produced by vortex pairing in a low Mach number jet," J. Sound Vib. 281, 171 (2005).

${ }^{43}$ V. Fleury, C. Bailly, E. Jondeau, M. Michard, and D. Juve, "Space-time correlation in two subsonic jets using dual Particle Image Velocimetry," AIAA J. 46, 2498 (2008).

${ }^{44}$ A. Henning, K. Kaepernick, K. Ehrenfried, L. Koop, and A. Dillmann, "Investigation of aeroacoustic noise generation by simultaneous velocimetry and microphone measurements," Exp. Fluids 45, 1073 (2008).

${ }^{45} \mathrm{C}$. Haigermoser, "Application of an acoustic analogy to PIV data from rectangular cavity flow," Exp. Fluids. 47, 145 (2009).
${ }^{46}$ V. Koschatzky, P. D. Moore, J. Westerweel, F. Scarano, and B. J. Boersma, "High-speed PIV applied to aerodynamic noise investigation," Exp. Fluids 50(4), 863 (2010).

${ }^{47}$ S. C. Morris, "Shear layer instabilities: Particle image velocimetry measurements and implications for acoustics," Annu. Rev. Fluid Mech. 43, 529 (2011).

${ }^{48}$ G. E. Elsinga, R. J. Adrian, B. W. van Oudheusden, and F. Scarano, "Three-dimensional vortex organization in a high-Reynolds-number supersonic turbulent boundary layer," J. Fluid Mech. 644, 35 (2010).

${ }^{49}$ A. Schroeder, R. Geisler, K. Staack, G. E. Elsinga, F. Scarano, B. Wieneke, A. Henning, C. Poelma, and J. Westerweel, "Eulerian and Lagrangian views of a turbulent boundary layer flow using time-resolved tomographic PIV," Exp. Fluids 50, 1071 (2010).

${ }^{50}$ D. Violato, P. Moore, and F. Scarano, "Lagrangian and Eulerian pressure field evaluation of rod-airfoil flow from time-resolved tomographic PIV," Exp. Fluids 50, 1057 (2011).

${ }^{51}$ F. Scarano and C. Poelma, "Three-dimensional vorticity patterns of cylinder wakes," Exp. Fluids 47, 69 (2009).

${ }^{52}$ F. Scarano, K. Bryon, and D. Violato, "Time resolved analysis of circular and chevron jets transition by TOMO-PIV," in 15th International Symposium on Applications of Laser Techniques to Fluid Mechanics, Lisbon, Portugal, 2010.

${ }^{53}$ D. Violato, P. Moore, K. Bryon, and F. Scarano, "Application of Powell's analogy for the prediction of vortex pairing sound in a low-mach number jet based on time resolved planar and tomographic PIV," in 16th AIAA/ CEAS Aeroacoustic Conference, Stockholm, Sweden, 2010.

${ }^{54}$ P. Moore, D. Violato, K. Bryon, and F. Scarano, "On the suitability of direct application of acoustic theory to time-resolved tomographic PIV tested by DNS for low mach number jet flows," in 16th AIAA/CEAS Aeroacoustic Conference, Stockholm, Sweden, 2010.

${ }^{55}$ J. Jeong and F. Hussain, "On the identification of a vortex," J. Fluid Mech. 285, 69 (1995).

${ }^{56}$ A. Powell, "Theory of vortex sound," J. Acoust. Soc. Am. 36, 177 (1964).

${ }^{57}$ G. T. Hermann and A. Lent, "Iterative reconstruction algorithms," Comput. Biol. Med. 6, 273 (1976).

${ }^{58} \mathrm{C}$. Willert, "Stereoscopic digital particle image velocimetry for application in wind tunnel flows," Meas. Sci. Technol. 8, 1465 (1997).

${ }^{59}$ B. Wieneke, "Volume self-calibration for 3D particle image velocimetry," Exp. Fluids 45, 549 (2008).

${ }^{60}$ C. D. Meinhart, S. T. Wereley, and J. G. Santiago, "A PIV algorithm for estimating time-averaged velocity fields," J. Fluids Eng. 122, 285 (2000).

${ }^{61}$ J. Westerweel, "Theoretical analysis of the measurement precision in particle image velocimetry," Exp. Fluids 29, 3 (2000).

${ }^{62}$ J. Sheng, E. Malkiel, and J. Katz, "Using digital holographic microscopy for simultaneous measurements of 3D near wall velocity and wall shear stress in a turbulent boundary layer," Exp. Fluids 45, 1023 (2008).

${ }^{63}$ F. Schrijer and F. Scarano, "Effect of predictor-corrector filtering on the stability and spatial resolution of iterative PIV interrogation," Exp. Fluids 45, 927 (2008).

${ }^{64}$ J. Zhang, B. Tao, and J. Katz, "Turbulent flow measurement in a square duct with hybrid holographic PIV," Exp. Fluids 285, 69 (1997).

${ }^{65}$ R. A. Petersen, "Influence of wave dispersion on vortex pairing in a jet," J. Fluid Mech. 89, 469 (1978).

${ }^{66}$ A. K. M. F. Hussain and K. B. M. Q. Zaman, "The preferred mode of the axisymmetric jet," J. Fluid Mech. 110, 39 (1981).

${ }^{67} \mathrm{G}$. B. Brown, "On vortex motion in gaseous jets and the origin of their sensitivity to sound," Proc. Phys. Soc. 47, 703 (1935).

${ }^{68}$ L. P. Bernal and A. Roshko, "Streamwise vortex structure in plane mixing layers," J. Fluid Mech. 170, 499 (1986).

${ }^{69}$ C. Dahan, G. Elias, J. Maulard, and M. Perulli, "Coherent structures in the mixing zone of a subsonic hot free jet," J. Sound Vib. 59(3), 313 (1978).

${ }^{70}$ P. Jordan, M. Schlegel, O. Stalnov, B. R. Noack, and C. E. Tinney, "Indentifying noisy and quiet modes in a jet," in 13th AIAA/CEAS Aeroacoustic Conference, Rome, Italy, 2007. 